\title{
Global well-posedness and large deviations for 3D stochastic Burgers equations
}

\author{
Rangrang Zhang ${ }^{1}$, Guoli Zhou ${ }^{2, *}$ Boling Guo ${ }^{3}$, Jiang-Lun $\mathrm{Wu}^{4}$, \\ ${ }^{1}$ School of Mathematics and Statistics, Beijing Institute of Technology, Beijing, 100081, China. \\ ${ }^{2}$ School of Statistics and Mathematics, Chongqing University, Chongqing, 400044, China \\ ${ }^{3}$ Institute of Applied Physics and Computational Mathematics, P.O. Box 8009, Beijing 100088, China \\ ${ }^{4}$ Department of Mathematics, Swansea University, Bay Campus Fabian Way, Swansea SA1 8EN Wales/UK \\ ( rrzhang@amss.ac.cn, zhouguoli736@126.com,gbl@iapcm.ac.cn,j.l.wu@swansea.ac.uk )
}

\begin{abstract}
In this paper, we study the stochastic vector-valued Burgers equations with non periodic boundary conditions. We first apply a contraction principle argument to show local existence and uniqueness of a mild solution to this model. Then, towards obtaining the global well-posedness, we derive a priori estimates of the local solution by utilising the maximum principle. Finally, we establish, by means of the weak convergence approach, the Freidlin-Wentzell type large deviation principle for 3D stochastic Burgers equations when the noise term goes to zero.
\end{abstract}

AMS Subject Classification: 60H15 76S05.

Keywords: 3D stochastic Burgers equations; global well-posedness; the Freidlin-Wentzell type large deviation principle.

\section{Introduction}

This paper is concerned with 3D positive viscosity stochastic Burgers equations prescribed on a bounded open domain $D \subset \mathbb{R}^{3}$. Fix any $T>0$ and let $\left(\Omega, \mathcal{F}, \mathbb{P},\left\{\mathcal{F}_{t}\right\}_{t \in[0, T]},\left(\left\{\beta_{k}(t)\right\}_{t \in[0, T]}\right)_{k \in \mathbb{N}}\right)$ be a stochastic basis. Without loss of generality, here the filtration $\left\{\mathcal{F}_{t}\right\}_{t \in[0, T]}$ is assumed to satisfy the usual conditions and $\left\{\beta_{k}(t)\right\}_{t \in[0, T]}, k \in \mathbb{N}$, are independent (one-dimensional) $\left\{\mathcal{F}_{t}\right\}_{t \in[0, T]}-$ Wiener processes. We use $\mathbb{E}$ to denote the expectation with respect to $\mathbb{P}$. We are concerned with the Cauchy problem for the 3D Burgers equations with stochastic forcing

$$
\begin{aligned}
& d u=(v \Delta u+(u \cdot \nabla) u) d t+G d W(t), \quad \text { on }[0, T] \times D, \\
& u(t, x)=0, \quad t \in[0, T], x=\left(x_{1}, x_{2}, x_{3}\right) \in \partial D \subset \mathbb{R}^{3}, \\
& u(0, x)=u_{0}(x), \quad x=\left(x_{1}, x_{2}, x_{3}\right) \in D \subset \mathbb{R}^{3},
\end{aligned}
$$

${ }^{*}$ Corresponding author. 
where the unknowns are the 3D vector-valued random fields $u(t, x)=\left(u_{1}(t, x), u_{2}(t, x), u_{3}(t, x)\right) \in \mathbb{R}^{3}, t \in$ $[0, T]$ and $x \in D$. The parameter $v>0$ in system (1.1) stands for the viscosity, $W(t)=\sum_{k \geq 1} \beta_{k}(t) e_{k}, t \in$ $[0, T]$, is a cylindrical Wiener process defined on a given (separable) Hilbert space $\mathbb{H}$ with $\left(e_{k}\right)_{k \geq 1}$ being a complete orthonormal base in $\mathbb{H}$, and the coefficient $G$ is a Hilbert-Schmidt operator which will be specified later. To simplify the notations, we set $\partial_{i}=\partial x_{i}, \quad i=1,2,3$. Moreover, $\Delta=\partial_{1}^{2}+\partial_{2}^{2}+\partial_{3}^{2}$ is the Laplace operator, $\nabla=\left(\partial_{1}, \partial_{2}, \partial_{3}\right)$ is the gradient operator.

The mathematical study of the Burgers equations were originated in a series of articles by Burgers (see [13]) in the early 1940s (and also in earlier papers by Beteman [6] and Forsyth [26]). As is known, the deterministic Burgers equations are not a suitable model to describe turbulence, since it does not perform any chaos even if a force is added to this model. Specifically, all the solutions to (deterministic) Burgers equations will converge to a unique stationary solution as time goes to infinity. However, when the force is changed to be a random one, the result is completely different, for details, see for instance $[14,18,32,30]$. In addition, the stochastic Burgers equations have also been applied to study the dynamics of interfaces in the work of [34]. One dimensional (i.e., scalar valued) stochastic Burgers equation has been fairly well studied. By an adaptation of the celebrated Hopf-Cole transformation, Bertini et al. [5] solved the one dimensional Burgers equation with additive space-time white noise, where the nonlinearity in the equation was formulated in Wick product. Moreover, Chan [15] utilised Hopf-Cole transformation to study the scaling limit of Wick ordered KPZ equation involving additive space-time white noise. Later, Da Prato et al. [18] studied the Burgers equation based on semigroup property for the heat equation on a bounded domain. E, Khanin, Mazel, and Sinai [24] establish the existence and uniqueness of a stationary distribution and obtained the convergence of stationary distributions in the limit when viscosity tends to zero. In [4], Bakhtin, Cator, and Khanin study the long-term behavior of the Burgers dynamics for the situation where the forcing is a space-time stationary random process. In particular, they construct space-time stationary global solutions for the Burgers equation on the real line and show that they can be viewed as one-point attractors. In [2], Bakhtin consider the Burgers equation with random boundary conditions. Concerning the one-dimensional Burgers equation with viscosity coefficient defined on a bounded domain driven by multiplicative Gaussian noise, Da Prato and Debussche [16] succeeded to obtain its global well-posedness. Furthermore, Gyöngy and Nualart [29] extended the results of Da Prato and Debussche to the Burgers equation defined on the whole line. By establishing the exponential tightness, Gourcy [27] show that a large deviation principle holds for the occupation measure of the solutions to the stochastic Burgers equations with small noises, which describes the exact rate of exponential convergence. When the Gaussian force is replaced by Lévy jumps, Dong and Xu proved its global well-posedness of the strong, weak and mild solutions as well as the ergodicity in [22, 20]. Later, Dong et al. generalized the noise to be $\alpha$-stable process and showed the exponential ergodicity in [21]. When the noise is changed to be space-time homogeneous random kick forcing, Bakhtin and Li [3] developed its ergodic theory without any compactness assumptions. Concretely, the authors proved a one force-one solution principle by applying the infinite-volume polymer measures to construct a family of stationary global solutions for this system, and proving that each of those solutions is a one point 
pullback attractor on the initial conditions with the same spatial average. When the initial condition for the Burgers equation is random, Mohammed and Zhang [37] showed the global well-posedness. Wang et al. [43] obtained the same result for the case that the noise is fractional Brownian motion. There are lots of other works which are very interesting and meaningful for the stochastic one dimensional Burgers equations. But here, we do not list them.

For the multidimensional Burgers equations, Kiselev and Ladyzhenskaya [35] proved the existence and uniqueness of solution in the class of functions $L^{\infty}\left(0, T ; L^{\infty}(O)\right) \cap L^{2}\left(0, T ; H_{0}^{1}(O)\right)$. When the viscosity $v \rightarrow 0$ and the initial condition is zero, Bui [12] proved the convergence of solutions to the inviscid Burgers equations on a small time interval. In the higher dimensional inviscid stochastic case, the stationary solution and a stationary distribution were constructed by Gomes, Iturriaga, Khanin and Padilla in [31] based on a very delicate use of the Lagrangian formalism and the Hamilton-Jacobi equation. Based on the stochastic version of Lax formula for solutions to the initial and final value problems for the viscous Hamilton-Jacobi equation, Gomes, Iturriaga, Khanin and Padilla in [28] prove convergence of stationary distributions for the randomly forced multi-dimensional Burgers and Hamilton-Jacobi equations in the limit when viscosity tends to zero. For the multidimensional generalised stochastic Burgers equation in the space-periodic setting, Boritchev [7] proved that if the solution $u$ of this equation is a gradient, then each of Sobolev norms of $u$ averaged in time and in ensemble behaves as a given negative power of the viscosity coefficient $\mu$, which gives the sharp upper and lower bounds for natural analogues of quantities characterising the hydrodynamical turbulence. Moreover, the author established the existence and uniqueness of stationary measure. Recently, Khanin and Zhang [33] generalized the results of [24] to arbitrary dimensional Burgers equation by using Green bundles method, which is complete different from the approach used by [24].

The purpose of this paper is two-fold. In the first part, we aim to show global well-posedness of 3D stochastic Burgers equations with non - periodic boundary conditions. Due to the dimensionality of this equation is 3 , the proof process is of great challenge. Our proof strategy mainly consists of the following procedures. Firstly, we change the stochastic Burgers equations into the random Burgers equations. Then using delicate and careful techniques in partial differential equations we establish a general frame to obtain the local existence and uniqueness for stochastic hydrodynamic systems. For the detail, one can see the Proposition 3.2. Here, we should mention that in [6], the local existence and uniqueness for the multi-dimensional stochastic Burgers equations are established in $C\left([0, T] ; L^{p}\left(D ; \mathbb{R}^{3}\right)\right)$, under the assumption the boundary conditions are periodic. At the present work the boundary conditions are nonperiodic, the local existence and uniqueness for the multi-dimensional stochastic Burgers equations are established in $C\left([0, T] ; \mathbb{H}^{1}\right)$ (see the notations in Section 2). In the following step, we try to prove the global well-posedness of stochastic 3D Burgers equations. A natural method is energy estimate. But we find that, the high dimensionality causes certain difficulties. For instance, when estimating the $L^{2}-$ norm of $v$, we encounter $\|v\|_{L^{4}}^{4}$, which can not be controlled by the dissipative term $\|\nabla v\|_{L^{2}}^{2}$. How to get around the difficulty, a nice method was given by [35], and then the method was extended to the stochastic case in [9]. Inspired by [9], we utilize the maximum and minimum principles to obtain the critical a priori 
estimates for the velocity fields. In this case, we need the assumption that the noise acts only in one coordinate to guarantee the application of these principles. Then, we succeed to obtain the $L^{\infty}$-estimates of the local mild solution in the both time and space variables. Based on it, we further obtain the uniform $\mathbb{H}^{1}$-norm of the local solution with respect to $t \in\left[0, T_{*}\right)$ where $T_{*}$ is the maximal existence time. Finally, we end up with the global existence of $u$. The second part is devoted to establishing the FreidlinWentzell's large deviation principle (LDP) for the 3D stochastic Burgers equations, which provides the exponential decay of small probabilities associated with the corresponding stochastic dynamical systems with small noise. A crucial tool for establishing the Freidlin-Wentzell's LDP is the weak convergence approach, which was developed by Dupuis and Ellis in [23]. The key idea of this approach is to prove some variational representation formulae about the Laplace transform of bounded continuous functionals, which will lead to the proof of equivalence between LDP and the Laplace principle. In particular, for Brownian functionals, an elegant variational representation formula has been established by Boué and Dupuis [8], Budhiraja and Dupuis [10]. As an important part of the proof, we need to obtain global well-posedness of the so called skeleton equation, whose global existence and uniqueness is proved by the same method as in the proof of Theorem 3.1. To complete the proof of the large deviation principle, we also study the weak convergence of the perturbations of the system (1.1) in the random directions of the Cameron-Martin space of the driving Brownian motions.

The paper is organized as follows. Some preliminaries are presented in Section 2, the local existence of the solutions and global well-posedness of 3D stochastic Burgers equations are given in Section 3 separately, the large deviations for 3D stochastic Burgers equations is established in Section 4. In the present paper, $C$ stands for a generic constant whose value may change from one line to the next, unless, we give a specific description. we denote by $C(a)$ a constant which depends on parameter $a$.

\section{Preliminaries}

Let us first introduce the notations which will be used later on. For $p \in \mathbb{Z}^{+}$, let $L^{p}\left(D ; \mathbb{R}^{3}\right)$ be the vectorvalued $L^{p}$-space in which the norm is denoted by $\|\cdot\|_{L^{p}}$. In particular, when $p=2$, denote by $H:=$ $L^{2}\left(D ; \mathbb{R}^{3}\right)$ and its associated norm and inner produce are $\|\cdot\|_{H}$ and $\langle\cdot, \cdot\rangle$, respectively. Moreover, when $p=\infty, L^{\infty}\left(D ; \mathbb{R}^{3}\right)$ denotes the collection of vector-valued functions which are essentially bounded on $D$ and the corresponding norm is denoted by $\|\cdot\|_{L_{x}^{\infty}}$.

For $m \in \mathbb{N}^{+},\left(W^{m, p}(D),\|\cdot\|_{m, p}\right)$ stands for the classical Sobolev space, see [1]. When $p=2$, we denote that $H^{m}(D)=W^{m, 2}(D)$,

$$
\|u\|_{H^{m}}^{2}=\sum_{0 \leq|\delta| \leq m} \int_{D}\left|D^{\delta} u\right|^{2} d x
$$

Here, $\delta$ is a multi-index, that is, $\delta=\left(\delta_{1}, \delta_{2}, \delta_{3}\right)$ with non-negative integers $\delta_{i}, i=1,2,3 .|\delta|=\sum_{i=1}^{3} \delta_{i}$. It's well-known that $\left(H^{m}(D),\|\cdot\|_{H^{m}}\right)$ is a Hilbert space. Let $C_{c}^{\infty}(D)$ be the space of all infinitely differentiable functions on $D$ with compact support. For $1 \leq p<\infty$, denote by $W_{0}^{1, p}(D)$ the closure of $C_{c}^{\infty}(D)$ in 
$W^{1, p}(D)$. Set $H_{0}^{1}(D)=W_{0}^{1,2}(D)$. Let $H^{-1}(D)$ be the dual space of $H_{0}^{1}(D)$.

\subsection{Reformulation}

Without loss of generality, we simply assume that the viscosity $v=1$. In fact, we only need $v$ to be any strictly positive number. Denote $A:=-\Delta$, then $A: H_{0}^{1}(D) \rightarrow H^{-1}(D)$ and $D(A)=\left[H^{2}(D) \cap\right.$ $\left.H_{0}^{1}(D)\right]^{3}$. It is well-known that $A$ is a positive, self-adjoint operator with discrete spectrum in $H$. Denote by $\left\{e_{n}\right\}_{n=1,2, \ldots}$ the eigenbasis of $A$ and further suppose that its associated eigenvalues $\left\{\alpha_{n}\right\}_{n=1,2, \ldots}$ is an increasing sequence. Moreover, we know that $\left\{e_{n}\right\}_{n=1,2, \cdots}$ form an orthonormal basis in $H$.

For any $s \in \mathbb{R}$, the fractional power $\left(A^{s}, D\left(A^{s}\right)\right)$ of the operator $(A, D(A))$ is defined as

$$
\left\{\begin{array}{l}
D\left(A^{s}\right)=\left\{u=\left.\sum_{n=1}^{\infty} u_{n} e_{n}\left|\sum_{n=1}^{\infty} \alpha_{n}^{2 s}\right| u_{n}\right|^{2}<\infty\right\} ; \\
A^{s} u=\sum_{n=1}^{\infty} \alpha_{n}^{s} u_{n} e_{n}, \quad \text { where } u=\sum_{n=1}^{\infty} u_{n} e_{n} .
\end{array}\right.
$$

Set

$$
\|u\|_{\mathbb{H}^{s}}=\left|A^{\frac{s}{2}} u\right|, \quad \mathbb{H}^{s}=D\left(A^{\frac{s}{2}}\right) .
$$

It is obvious that $\left(\mathbb{H}^{s},\|\cdot\|_{\mathbb{H}^{s}}\right)$ is a Hilbert space, and that $\left(\mathbb{H}^{0},\|\cdot\|_{\mathbb{H}^{0}}\right)=\left(H,\|\cdot\|_{H}\right)$. Moreover, thanks to the regularity theory of the Stokes operator, $\mathbb{H}^{s}$ is a closed subspace of $H^{s}\left(D ; \mathbb{R}^{3}\right)$ and $\|\cdot\|_{\mathbb{H}^{s}}$ is equivalent to the usual norm of $H^{s}\left(D ; \mathbb{R}^{3}\right)$. For simplicity, denote by $\mathbb{H}=\mathbb{H}_{0}=H$. It's obvious that $\left(\mathbb{H}^{s},\|\cdot\|_{\mathbb{H}^{s}}\right)$ is a Hilbert space.

Define the bilinear operator $B(u, v): \mathbb{H}^{1} \times \mathbb{H}^{1} \rightarrow \mathbb{H}^{-1}$ as

$$
\langle B(u, v), z\rangle=\int_{D} z(x) \cdot(u(x) \cdot \nabla) v(x) d x
$$

for all $z \in \mathbb{H}^{1}$. Based on the above, (1.1) can be written as follows

$$
d u(t)+A u(t) d t=B(u(t), u(t)) d t+G d W(t) .
$$

\subsection{Hypotheses}

As stated in the introduction, we first establish the existence and uniqueness of local solutions to 3D Burgers equations. To this end, we need the following hypothesis.

Hypothesis H0 $W$ is a cylindrical Wiener process in $\mathbb{H}$ defined on $(\Omega, \mathcal{F}, \mathbb{P})$ with the form $W(t)=$ $\sum_{k \geq 1} \beta_{k}(t) e_{k}, t \in[0, T]$, where $\left(e_{k}\right)_{k \geq 1}$ is a complete orthonormal base in $\mathbb{H}$. We assume that $G W(t)$ has the following representative:

$$
G W(t, x)=\sum_{k=1}^{\infty} \sqrt{\lambda_{k}} e_{k}(x) \beta_{k}(t) \in \mathbb{R}^{3} \text {, a.e. } x \in D,
$$

and there exists a constant $\varsigma \in\left(0, \frac{1}{2}\right)$ such that $\sum_{k=1}^{\infty} \lambda_{k} \alpha_{k}^{\frac{3}{2}+2 \varsigma}<\infty$. That is, $G$ is a Hilbert-Schmidt operator from $\mathbb{H}$ to $\left(\mathbb{H}^{\frac{3}{2}+2 \varsigma}\right)^{3}$. 
Next, in order to obtain the global existence of solutions to 3D Burgers equations, we need a stronger condition on the noise. Actually, we require that the noise acts only on one coordinate to ensure that the maximal and minimum principles are applicable to 3D Burgers equations. Specifically,

Hypothesis $\mathbf{H 1}$ Denote by $\left\{\bar{e}_{k}\right\}_{k=1,2, \cdots}$ the eigenbasis of $A$ in the space $L^{2}\left(D ; \mathbb{R}^{1}\right)$ and its associated eigenvalues is still denoted by $\left\{\alpha_{k}\right\}_{k=1,2, \cdots}$ Let $r_{k}:=\left(\bar{e}_{k}, 0,0\right), k=1,2, \cdots$, and for any $t \in[0, T]$, we assume that $W(t)=\sum_{k \geq 1} \beta_{k}(t) r_{k}, t \in[0, T]$ and

$$
G W(t, x)=\sum_{k=1}^{\infty} \sqrt{\lambda_{k}} r_{k}(x) \beta_{k}(t)=\left(\sum_{k=1}^{\infty} \sqrt{\lambda_{k}} \bar{e}_{k}(x) \beta_{k}(t), 0,0\right) \in \mathbb{R}^{3} \text {, a.e. } x \in D .
$$

\subsection{An auxiliary process}

Consider the following auxiliary equation:

$$
\begin{aligned}
d Z(t)+A Z d t & =G d W(t), \quad \text { on }[0, T] \times D, \\
Z(t, x) & =0, \quad t \in[0, T], x \in \partial D,
\end{aligned}
$$

with the initial value $Z(0, x)=0$. It can be derived from [17] that (2.4) has a unique solution

$$
Z(t)=\int_{0}^{t} e^{-(t-s) A} G d W(s)
$$

By Theorem 5.20 in [17], we know that $Z(t)=Z(t, \cdot)$ is Gaussian process taking values in $\mathbb{H}$ and for any $T>0$, it has a version $Z(t, x)$, which is, a.s. for $\omega \in \Omega, \alpha$-Hölder continuous with respect to $(t, x)$, for any $\alpha \in\left[0, \frac{1}{4}\right.$ ) (see Theorem 5.20 in [17]). In addition, it holds that

Proposition 2.1. Under Hypothesis $H 0$, for any $p, T>0$, there exists a positive constant $C_{p, T}$ such that

$$
\mathbb{E} \sup _{t \in[0, T]}\|Z(t)\|_{\mathbb{H}^{\frac{5}{2}}}^{2 p} \leq C_{p, T}<\infty
$$

that is, the process $Z(t)$ takes values in $\mathbb{H}^{\frac{5}{2}}, \mathbb{P}$-a.s. $\omega \in \Omega$.

Proof. By the Hölder inequality, it suffices to prove (2.5) holds for large $p$. Using the factorization method, for $\varsigma \in\left(0, \frac{1}{2}\right)$ in Hypothesis H0, it holds that

$$
Z(t)=\frac{\sin \pi \varsigma}{\pi} \int_{0}^{t} e^{-(t-s) A}(t-s)^{\varsigma^{-1}} Y_{s} d s,
$$

where

$$
Y_{s}=\int_{0}^{s} e^{-(s-r) A}(s-r)^{-\varsigma} G d W(r) .
$$

Choosing $p>1$ large enough such that $\frac{2 p(1-\varsigma)}{2 p-1}<1$, then $\varsigma>\frac{1}{2 p}$. By Hölder inequality, we get

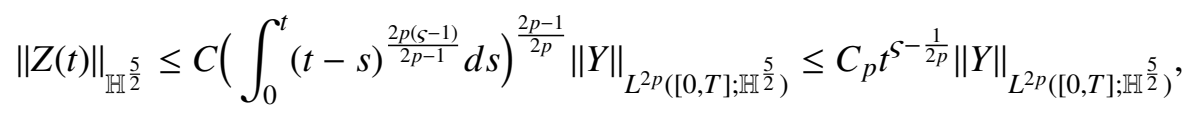


which implies

$$
\sup _{t \in[0, T]}\|Z(t)\|_{\mathbb{H}^{\frac{5}{2}}}^{2 p} \leq C_{p, T}\|Y\|_{L^{2 p}\left([0, T] ; \mathbb{H}^{\frac{5}{2}}\right)}^{2 p}
$$

Notice that $A^{\frac{5}{4}} Y_{S} \sim N\left(0, \tilde{Q}_{s}\right)$, which is a Gaussian random variable with mean zero and the covariance operator given by

$$
\tilde{Q}_{s} x=\int_{0}^{s} r^{-2} e^{-r A} A^{\frac{5}{2}} G G^{*} e^{-r A^{*}} x d r .
$$

For any $p \geq 1, s>0$, referring to Corollary 2.17 in [17], it gives

$$
\begin{aligned}
\mathbb{E}\left\|A^{\frac{5}{4}} Y_{s}\right\|^{2 p} & \leq C_{p}\left|\operatorname{Tr}\left(\tilde{Q}_{s}\right)\right|^{p}=C_{p}\left[\sum_{k=1}^{\infty}\left\langle\tilde{Q}_{s} e_{k}, e_{k}\right\rangle\right]^{p}=C_{p}\left[\sum_{k=1}^{\infty} \int_{0}^{s} r^{-2 \varsigma} e^{-2 r \alpha_{k}} \alpha_{k}^{\frac{5}{2}} \lambda_{k} d r\right]^{p} \\
& =C_{p}\left[\sum_{k=1}^{\infty} \alpha_{k}^{\frac{5}{2}+2 \varsigma-1} \lambda_{k} \int_{0}^{2 s \alpha_{k}} r^{-2 \varsigma} e^{-r} d r\right]^{p} \leq C_{p}\left[\sum_{k=1}^{\infty} \alpha_{k}^{\frac{3}{2}+2 \varsigma} \lambda_{k} \Gamma(-2 \varsigma+1)\right]^{p} \\
& \leq C_{p}\left[\sum_{k=1}^{\infty} \alpha_{k}^{\frac{3}{2}+2 \varsigma} \lambda_{k}\right]^{p}<\infty
\end{aligned}
$$

where we have used Hypothesis H0. Finally, we conclude that

$$
\mathbb{E} \sup _{t \in[0, T]}\|Z(t)\|_{\mathbb{H}^{\frac{5}{2}}}^{2 p} \leq C_{p, T} \mathbb{E}\|Y\|_{L^{2 p}\left([0, T] ; \mathbb{H}^{\frac{5}{2}}\right)}^{2 p} \leq C_{p, T}\left[\sum_{k=1}^{\infty} \alpha_{k}^{\frac{3}{2}+2 \varsigma} \lambda_{k}\right]^{p}<\infty .
$$

\section{Global well-posedness of 3D Burgers equations}

In this part, we devote to establishing global well-posedness of 3D Burgers equations.

Definition 3.1. For any $T>0$, a stochastic process $u(t)$ is called a mild solution to $(2.2)$ in $[0, T]$, if for $\mathbb{P}-$ a.s. $\omega \in \Omega$, u satisfies

$$
u(t)=e^{-t A} u_{0}+\int_{0}^{t} e^{-(t-s) A} B(u, u) d s+\int_{0}^{t} e^{-(t-s) A} G d W(s), \forall t \in[0, T] .
$$

Moreover, $u \in C\left([0, T] ; \mathbb{H}^{1}\right) \cap L^{2}\left([0, T] ; \mathbb{H}^{2}\right)$

Referring to Proposition 2.1 and Proposition 2.2 in [17], the mild solution to (2.2) is equivalent to the following strong solution.

Definition 3.2. An $\left(\mathcal{F}_{t}\right)_{t \geq 0}$-adapted process $(u(t))_{t \in[0, T]}$ is called a strong solution to (2.2) if $\mathbb{P}$-a.s. $\omega \in \Omega,(u(t))_{t \in[0, T]} \in C\left([0, T] ; \mathbb{H}^{1}\right) \cap L^{2}\left([0, T] ; \mathbb{H}^{2}\right)$, and satisfies

$$
u(t)+\int_{0}^{t} A u(s) d s=u(0)+\int_{0}^{t} B(u, u)(s) d s+\int_{0}^{t} G d W(s), \mathbb{P}-\text { a.s. }
$$

for all $t \in[0, T]$. 
Let $v(t)=u(t)-Z(t)$. A stochastic process $u(t, \omega)$ is a solution to (2.2) on $[0, T]$, if and only if $v$ is a solution to the following problem on $[0, T]$ :

$$
\begin{aligned}
d v+A v d t & =B(v+Z, v+Z) d t, \quad \text { on }[0, T] \times D, \\
v(t, x) & =0, \quad t \in[0, T], x \in \partial D
\end{aligned}
$$

with initial value $v(0)=u_{0}(x)$.

Definition 3.3. Let $Z$ be defined by (2.4) and $T>0$. For $\mathbb{P}-$ a.s. $\omega \in \Omega$, $v$ is called a mild solution to (3.6) on the time interval $[0, T]$, if it belongs to $C\left([0, T] ; \mathbb{H}^{1}\right) \cap L^{2}\left([0, T] ; \mathbb{H}^{2}\right)$ and satisfies

$$
v(t)=e^{-t A} u_{0}+\int_{0}^{t} e^{-(t-s) A} B(v+Z, v+Z) d s, \quad t \in[0, T] .
$$

Our first main result of the present paper reads as follows

Theorem 3.1. For any $\mathcal{F}_{0}$-adapted initial value $u_{0}$ satisfying $\left\|u_{0}\right\|_{L_{x}^{\infty}} \vee\left\|u_{0}\right\|_{\mathbb{H}^{1}}<\infty$ and any $T>0$, under Hypotheses $\mathrm{HO}$ and $\mathrm{H} 1$, there exists a unique global solution $(u(t))_{t \in[0, T]}$ to $(2.2)$ in the sense of Definition 3.2. Furthermore, the solution $(u(t))_{t \in[0, T]}$ to (2.2) is Lipschitz continuous with respect to the initial data in $\mathbb{H}^{1}$.

Based on the above discussion, in order to prove Theorem 3.1, it suffices to prove the global wellposedness of (3.6) in the sense of Definition 3.3. To achieve this, we first prove that for $\mathbb{P}-$ a.s. $\omega \in \Omega$, there exists a small positive constant $T_{*}(\omega)$ and a unique mild solution $v$ to (3.6) on a short time interval $\left[0, T_{*}(\omega)\right)$. Then, by establishing uniform a priori estimates of this solution, we show the maximal existence time $\tau_{*}=+\infty, \mathbb{P}-$ a.s..

\subsection{Local existence and uniqueness of the strong solutions}

In the sequel, $\omega \in \Omega$ is fixed. We shall prove the local existence of a mild solution to (3.6) by applying the classical fixed point theorem.

Proposition 3.2. Assume $u_{0}$ is $\mathcal{F}_{0}$-adapted initial value satisfying $\left\|u_{0}\right\|_{\mathbb{H}^{1}}<\infty$ and Hypothesis $H 0$ is in force, then for any $T>0$ and $\mathbb{P}$-a.s. $\omega \in \Omega$, there exists $0<T_{*}(\omega)<T$ and $v$ such that $v$ is a unique mild solution to (3.6) with the initial condition $u_{0}$ on the time interval $\left[0, T_{*}(\omega)\right)$.

Proof. For arbitrarily fixed $T>0$, let $v$ be an $(\mathcal{F}(t))_{t \geq 0}$-adapted process such that $v \in C\left([0, T] ; \mathbb{H}^{1}\right)$ and define a mapping

$$
\mathcal{L}(v):=e^{-t A} u_{0}+\int_{0}^{t} e^{-(t-s) A}[(v+Z) \cdot \nabla](v+Z) d s, \quad t \in[0, T] .
$$

We will show that $\mathcal{L}$ is a contraction mapping in the following space:

$$
\mathcal{B}_{R}^{T_{*}}=\left\{v \in C\left(\left[0, T_{*}\right) ; \mathbb{H}^{1}\right): \sup _{t \in\left[0, T_{*}\right)}\|v(t)\|_{\mathbb{H}^{1}}+\sup _{t \in\left[0, T_{*}\right)} t^{\frac{7}{13}}\|v(t)\|_{\mathbb{H}^{2}} \leq R, v(0)=u_{0}\right\},
$$


where

$$
R:=3\left(\sup _{t \in[0, T]}\|Z\|_{\mathbb{H}^{2}}+\left\|u_{0}\right\|_{\mathbb{H}^{1}}\right)
$$

and $T_{*}<T$ will be chosen sufficiently small. Clearly, $R$ and $T_{*}$ depend on $\omega \in \Omega$.

For any $v \in \mathcal{B}_{R}^{T_{*}}$ and $0 \leq t \leq T_{*}$, by Theorem 6.13 in [39], it yields

$$
\begin{aligned}
\|\mathcal{L}(v)\|_{\mathbb{H}^{1}} & \leq\left\|e^{-t A} u_{0}\right\|_{\mathbb{H}^{1}}+\int_{0}^{t}\left\|e^{-(t-s) A}((v+Z) \cdot \nabla)(v+Z)\right\|_{\mathbb{H}^{1}} d s \\
& \leq\left\|u_{0}\right\|_{\mathbb{H}^{1}}+\int_{0}^{t}(t-s)^{-\frac{1}{2}}\|((v+Z) \cdot \nabla)(v+Z)\|_{\mathbb{H}} d s \\
& \leq\left\|u_{0}\right\|_{\mathbb{H}^{1}}+\int_{0}^{t}(t-s)^{-\frac{1}{2}}\|v+Z\|_{L_{x}^{\infty}}\|\nabla(v+Z)\|_{\mathbb{H}} d s .
\end{aligned}
$$

By Agmon's inequality, we have

$$
\|u\|_{L_{x}^{\infty}} \leq C\|u\|_{\mathbb{H}^{1}}^{\frac{1}{2}}\|u\|_{\mathbb{H}^{2}}^{\frac{1}{2}},
$$

where $C$ is a positive constant independent of $t \in[0, T]$. In the rest article, $C$ is a positive constant which is independent of time $t$ unless it is given a specific description. Hence, for $t \in\left[0, T_{*}\right)$, it follows that

$$
\begin{aligned}
\|\mathcal{L}(v)\|_{\mathbb{H}^{1}} & \leq\left\|u_{0}\right\|_{\mathbb{H}^{1}}+C \int_{0}^{t}(t-s)^{-\frac{1}{2}}\|v+Z\|_{\mathbb{H}^{1}}^{\frac{1}{2}}\|v+Z\|_{\mathbb{H}^{2}}^{\frac{1}{2}}\|\nabla(v+Z)\|_{L^{2}} d s \\
& \leq\left\|u_{0}\right\|_{\mathbb{H}^{1}}+C \int_{0}^{t}(t-s)^{-\frac{1}{2}} s^{-\frac{7}{26}}\|v+Z\|_{\mathbb{H}^{1}}^{\frac{3}{2}}\left(s^{\frac{7}{13}}\|v+Z\|_{\mathbb{H}^{2}}\right)^{\frac{1}{2}} d s \\
& \leq \frac{R}{3}+C R^{2} \int_{0}^{t}(t-s)^{-\frac{1}{2}} s^{-\frac{7}{26}} d s+C R^{2} \int_{0}^{t}(t-s)^{-\frac{1}{2}} d s \\
& \leq \frac{R}{3}+C R^{2}\left(t^{\frac{3}{13}}+t^{\frac{1}{2}}\right) .
\end{aligned}
$$

For $0 \leq t \leq T$, by Theorem 6.13 in [39], we deduce that

$$
\begin{aligned}
t^{\frac{7}{13}}\|\mathcal{L}(v)\|_{\mathbb{H}^{2}} & \leq t^{\frac{7}{13}}\left\|e^{-A t} u_{0}\right\|_{\mathbb{H}^{2}}+t^{\frac{7}{13}} \int_{0}^{t}\left\|e^{-A(t-s)}((v+Z) \cdot \nabla)(v+Z)\right\|_{\mathbb{H}^{2}} d s \\
& \leq t^{\frac{1}{26}}\left\|u_{0}\right\|_{\mathbb{H}^{1}}+t^{\frac{7}{13}} \int_{0}^{t}(t-s)^{-\frac{1}{2}}\|((v+Z) \cdot \nabla)(v+Z)\|_{\mathbb{H}^{1}} d s \\
& \leq t^{\frac{1}{26}}\left\|u_{0}\right\|_{\mathbb{H}^{1}}+t^{\frac{7}{13}} \int_{0}^{t}(t-s)^{-\frac{1}{2}}\left(\|\nabla u\|_{L^{4}}^{2}+\|u\|_{L_{x}^{\infty}}\|u\|_{\mathbb{H}^{2}}\right) d s .
\end{aligned}
$$

By the Gagliardo-Nirenberg interpolation inequalities(see [41]), it gives

$$
\|u\|_{L^{4}} \leq C\|u\|_{\mathbb{H}^{\frac{1}{4}}}^{\frac{1}{4}}\|u\|_{\mathbb{H}^{1}}^{\frac{3}{4}} .
$$

With the help of (3.7) and (3.9), it follows that

$$
\begin{aligned}
t^{\frac{7}{13}}\|\mathcal{L}(v)\|_{\mathbb{H}^{2}} & \leq t^{\frac{1}{26}}\left\|u_{0}\right\|_{\mathbb{H}^{1}}+C t^{\frac{7}{13}} \int_{0}^{t}(t-s)^{-\frac{1}{2}}\|v+Z\|_{\mathbb{H}^{1}}^{\frac{1}{2}}\|v+Z\|_{\mathbb{H}^{2}}^{\frac{3}{2}} d s \\
& \leq t^{\frac{1}{26}} \frac{R}{3}+C R^{\frac{1}{2}} t^{\frac{7}{13}} \int_{0}^{t}(t-s)^{-\frac{1}{2}}\left(s^{-\frac{7}{13}} s^{\frac{7}{13}}\|v\|_{\mathbb{H}^{2}}\right)^{\frac{3}{2}} d s+C R^{2} t^{\frac{7}{13}} \int_{0}^{t}(t-s)^{-\frac{1}{2}} d s \\
& \leq t^{\frac{1}{26}} \frac{R}{3}+C R^{2}\left(t^{\frac{3}{13}}+t^{\frac{27}{26}}\right) .
\end{aligned}
$$


Combining (3.8) and (3.10), we get

$$
\|\mathcal{L}(v)\|_{\mathbb{H}^{1}}+t^{\frac{7}{13}}\|\mathcal{L}(v)\|_{\mathbb{H}^{2}} \leq \frac{R}{3}+C\left(R+R^{2}\right)\left(t^{\frac{1}{26}}+t^{\frac{1}{2}}+t^{\frac{3}{13}}+t^{\frac{27}{26}}\right) .
$$

For $v_{1}, v_{2} \in B_{R}^{T_{*}}$, it follows that

$$
\mathcal{L}\left(v_{1}\right)-\mathcal{L}\left(v_{2}\right)=\int_{0}^{t} e^{-(t-s) A}\left[\left(\left(v_{1}+Z\right) \cdot \nabla\right)\left(v_{1}+Z\right)-\left(\left(v_{2}+Z\right) \cdot \nabla\right)\left(v_{2}+Z\right)\right] d s .
$$

By utilizing Theorem 6.13 again in [39], it yields

$$
\begin{aligned}
\left\|\mathcal{L}\left(v_{1}\right)-\mathcal{L}\left(v_{2}\right)\right\|_{\mathbb{H}^{1}} \leq & \int_{0}^{t}\left\|e^{-(t-s) A}\left[\left(\left(v_{1}+Z\right) \cdot \nabla\right)\left(v_{1}+Z\right)-\left(\left(v_{2}+Z\right) \cdot \nabla\right)\left(v_{2}+Z\right)\right]\right\|_{\mathbb{H}^{1}} d s \\
\leq & \int_{0}^{t}(t-s)^{-\frac{1}{2}}\left\|\left[\left(\left(v_{1}+Z\right) \cdot \nabla\right)\left(v_{1}+Z\right)-\left(\left(v_{2}+Z\right) \cdot \nabla\right)\left(v_{2}+Z\right)\right]\right\|_{\mathbb{H}^{1}} d s \\
\leq & \int_{0}^{t}(t-s)^{-\frac{1}{2}}\left\|\left(\left(v_{1}+Z\right) \cdot \nabla\right)\left(v_{1}-v_{2}\right)\right\|_{\mathbb{H}} d s \\
& +\int_{0}^{t}(t-s)^{-\frac{1}{2}}\left\|\left(\left(v_{1}-v_{2}\right) \cdot \nabla\right)\left(v_{2}+Z\right)\right\|_{\mathbb{H}} d s \\
\leq & \int_{0}^{t}(t-s)^{-\frac{1}{2}}\left\|v_{1}+Z\right\|_{L_{x}^{\infty}}\left\|v_{1}-v_{2}\right\|_{\mathbb{H}^{1}} d s \\
& +\int_{0}^{t}(t-s)^{-\frac{1}{2}}\left\|v_{1}-v_{2}\right\|_{L^{4}}\left\|\nabla\left(v_{2}+Z\right)\right\|_{L^{4}} d s .
\end{aligned}
$$

Using (3.7) and (3.9), we get

$$
\begin{aligned}
\left\|\mathcal{L}\left(v_{1}\right)-\mathcal{L}\left(v_{2}\right)\right\|_{\mathbb{H}^{1}} \leq & C \int_{0}^{t}(t-s)^{-\frac{1}{2}}\left\|v_{1}+Z\right\|_{\mathbb{H}^{1}}^{\frac{1}{2}}\left\|v_{1}+Z\right\|_{\mathbb{H}^{2}}^{\frac{1}{2}}\left\|v_{1}-v_{2}\right\|_{\mathbb{H}^{1}} d s \\
& +C \int_{0}^{t}(t-s)^{-\frac{1}{2}}\left\|v_{2}+Z\right\|_{\mathbb{H}^{1}}^{\frac{1}{4}}\left\|v_{2}+Z\right\|_{\mathbb{H}^{2}}^{\frac{3}{4}}\left\|v_{1}-v_{2}\right\|_{\mathbb{H}^{1}} d s \\
=: & I_{1}(t)+I_{2}(t) .
\end{aligned}
$$

We begin with the estimates of $I_{1}$, it yields

$$
\begin{aligned}
I_{1}(t) & \leq C \int_{0}^{t}(t-s)^{-\frac{1}{2}} R^{\frac{1}{2}}\left(R+s^{-\frac{7}{13}} s^{\frac{7}{13}}\left\|v_{1}\right\|_{\mathbb{H}^{2}}\right)^{\frac{1}{2}}\left\|v_{1}-v_{2}\right\|_{\mathbb{H}^{1}} d s \\
& \leq C \int_{0}^{t}(t-s)^{-\frac{1}{2}} R\left(1+s^{-\frac{7}{26}}\right)\left\|v_{1}-v_{2}\right\|_{\mathbb{H}^{1}} d s \\
& \leq C R \int_{0}^{t}(t-s)^{-\frac{1}{2}}\left\|v_{1}-v_{2}\right\|_{\mathbb{H}^{1}} d s+C R \int_{0}^{t}(t-s)^{-\frac{1}{2}} s^{-\frac{7}{26}}\left\|v_{1}-v_{2}\right\|_{\mathbb{H}^{1}} d s \\
& \leq C R\left(t^{\frac{1}{2}}+t^{\frac{3}{13}}\right) \sup _{t \in\left[0, T_{*}\right)}\left\|v_{1}-v_{2}\right\|_{\mathbb{H}^{1}} .
\end{aligned}
$$

Similarly, we have

$$
I_{2}(t) \leq C R\left(t^{\frac{1}{2}}+t^{\frac{5}{52}}\right) \sup _{t \in\left[0, T_{*}\right)}\left\|v_{1}-v_{2}\right\|_{\mathbb{H}^{1}}
$$


Combining (3.12) and (3.13), we conclude that

$$
\left\|\mathcal{L}\left(v_{1}\right)-\mathcal{L}\left(v_{2}\right)\right\|_{\mathbb{H}^{1}} \leq C R\left(t^{\frac{1}{2}}+t^{\frac{3}{13}}+t^{\frac{5}{52}}\right) \sup _{t \in\left[0, T_{*}\right)}\left\|v_{1}-v_{2}\right\|_{\mathbb{H}^{1}} .
$$

Moreover, by Theorem 6.13 in [39], we get

$$
\begin{aligned}
t^{\frac{7}{13}}\left\|\mathcal{L}\left(v_{1}\right)-\mathcal{L}\left(v_{2}\right)\right\|_{\mathbb{H}^{2}} \leq & t^{\frac{7}{13}} \int_{0}^{t}\left\|e^{-(t-s) A}\left(\left(v_{1}+Z\right) \cdot \nabla\right)\left(v_{1}+Z\right)-\left(\left(v_{2}+Z\right) \cdot \nabla\right)\left(v_{2}+Z\right)\right\|_{\mathbb{H}^{2}} d s \\
\leq & t^{\frac{7}{13}} \int_{0}^{t}(t-s)^{-\frac{1}{2}}\left\|\left(\left(v_{1}+Z\right) \cdot \nabla\right)\left(v_{1}+Z\right)-\left(\left(v_{2}+Z\right) \cdot \nabla\right)\left(v_{2}+Z\right)\right\|_{\mathbb{H}^{1}} d s \\
\leq & t^{\frac{7}{13}} \int_{0}^{t}(t-s)^{-\frac{1}{2}}\left\|\left(\left(v_{1}-v_{2}\right) \cdot \nabla\right)\left(v_{1}+Z\right)\right\|_{\mathbb{H}^{1}} d s \\
& +t^{\frac{7}{13}} \int_{0}^{t}(t-s)^{-\frac{1}{2}}\left\|\left(\left(v_{2}+Z\right) \cdot \nabla\right)\left(v_{1}-v_{2}\right)\right\|_{\mathbb{H}^{1}} d s \\
= & I_{3}(t)+I_{4}(t) .
\end{aligned}
$$

For $I_{3}$, by (3.7) and (3.9), we deduce that

$$
\begin{aligned}
& I_{3}(t) \leq t^{\frac{7}{13}} \int_{0}^{t}(t-s)^{-\frac{1}{2}}\left\|v_{1}-v_{2}\right\|_{L_{x}^{\infty}}\left\|v_{1}+Z\right\|_{\mathbb{H}^{2}} d s \\
& +t^{\frac{7}{13}} \int_{0}^{t}(t-s)^{-\frac{1}{2}}\left\|\nabla\left(v_{1}+Z\right)\right\|_{L^{4}}\left\|\nabla\left(v_{1}-v_{2}\right)\right\|_{L^{4}} d s \\
& \leq C t^{\frac{7}{13}} \int_{0}^{t}(t-s)^{-\frac{1}{2}}\left\|v_{1}-v_{2}\right\|_{\mathbb{H}^{1}}^{\frac{1}{2}}\left\|v_{1}-v_{2}\right\|_{\mathbb{H}^{2}}^{\frac{1}{2}}\left\|v_{1}+Z\right\|_{\mathbb{H}^{2}} d s \\
& +t^{\frac{7}{13}} \int_{0}^{t}(t-s)^{-\frac{1}{2}}\left\|\nabla\left(v_{1}+Z\right)\right\|_{\mathbb{H}}^{\frac{1}{4}}\left\|\nabla\left(v_{1}+Z\right)\right\|_{\mathbb{H}^{1}}^{\frac{3}{4}}\left\|\nabla\left(v_{1}-v_{2}\right)\right\|_{\mathbb{H}^{1}}^{\frac{1}{4}}\left\|\nabla\left(v_{1}-v_{2}\right)\right\|_{\mathbb{H}^{1}}^{\frac{3}{4}} d s \\
& \leq C t^{\frac{7}{13}} \int_{0}^{t}(t-s)^{-\frac{1}{2}} s^{-\frac{21}{26}}\left\|v_{1}-v_{2}\right\|_{\mathbb{H}^{1}}^{\frac{1}{2}}\left(s^{\frac{7}{13}}\left\|v_{1}-v_{2}\right\|_{\mathbb{H}^{2}}\right)^{\frac{1}{2}}\left(s^{\frac{7}{13}}\left\|v_{1}+Z\right\|_{\mathbb{H}^{2}}\right) d s \\
& +C t^{\frac{7}{13}} \int_{0}^{t}(t-s)^{-\frac{1}{2}} s^{-\frac{21}{26}}\left\|v_{1}+Z\right\|_{\mathbb{H}^{1}}^{\frac{1}{4}}\left(s^{\frac{7}{13}}\left\|v_{1}+Z\right\|_{\mathbb{H}^{2}}\right)^{\frac{3}{4}}\left\|v_{1}-v_{2}\right\|_{\mathbb{H}^{1}}^{\frac{1}{4}}\left(s^{\frac{7}{13}}\left\|v_{1}-v_{2}\right\|_{\mathbb{H}^{2}}\right)^{\frac{3}{4}} d s \\
& \leq C R t^{\frac{7}{13}} \int_{0}^{t}(t-s)^{-\frac{1}{2}} s^{-\frac{21}{26}} d s\left(\sup _{t \in\left[0, T_{*}\right)}\left\|v_{1}-v_{2}\right\|_{\mathbb{H}^{1}}+\sup _{t \in\left[0, T_{*}\right)} t^{\frac{7}{13}}\left\|v_{1}-v_{2}\right\|_{\mathbb{H}^{2}}\right) \\
& \leq C R t^{\frac{3}{13}}\left(\sup _{t \in\left[0, T_{*}\right)}\left\|v_{1}-v_{2}\right\|_{\mathbb{H}^{1}}+\sup _{t \in\left[0, T_{*}\right)} t^{\frac{7}{13}}\left\|v_{1}-v_{2}\right\|_{\mathbb{H}^{2}}\right) \text {. }
\end{aligned}
$$

Similarly, it follows that

$$
I_{4}(t) \leq C R\left(t^{\frac{33}{52}}+t^{\frac{27}{26}}\right) \sup _{t \in\left[0, T_{*}\right)}\left\|v_{1}-v_{2}\right\|_{\mathbb{H}^{1}}+C R\left(t^{\frac{5}{52}}+t^{\frac{3}{13}}+t^{\frac{1}{2}}\right) \sup _{t \in\left[0, T_{*}\right)} t^{\frac{7}{13}}\left\|v_{1}-v_{2}\right\|_{\mathbb{H}^{2}}
$$

In view of (3.15) and (3.16), we conclude that

$$
\begin{aligned}
t^{\frac{7}{13}}\left\|\mathcal{L}\left(v_{1}\right)-\mathcal{L}\left(v_{2}\right)\right\|_{\mathbb{H}^{2}} \leq & C R\left(t^{\frac{3}{13}}+t^{\frac{33}{52}}+t^{\frac{27}{26}}\right) \sup _{t \in\left[0, T_{*}\right)}\left\|v_{1}-v_{2}\right\|_{\mathbb{H}^{1}} \\
& +C R\left(t^{\frac{5}{52}}+t^{\frac{3}{13}}+t^{\frac{1}{2}}\right) \sup _{t \in\left[0, T_{*}\right)} t^{\frac{7}{13}}\left\|v_{1}-v_{2}\right\|_{\mathbb{H}^{2}} .
\end{aligned}
$$


As a result, combining (3.14) and (3.17), it yields

$$
\begin{aligned}
& \left\|\mathcal{L}\left(v_{1}\right)-\mathcal{L}\left(v_{2}\right)\right\|_{\mathbb{H}^{1}}+t^{\frac{7}{13}}\left\|\mathcal{L}\left(v_{1}\right)-\mathcal{L}\left(v_{2}\right)\right\|_{\mathbb{H}^{2}} \\
\leq & C R\left(t^{\frac{5}{52}}+t^{\frac{3}{13}}+t^{\frac{1}{2}}+t^{\frac{33}{52}}+t^{\frac{27}{26}}\right)\left(\sup _{t \in\left[0, T_{*}\right)}\left\|v_{1}-v_{2}\right\|_{\mathbb{H}^{1}}\right) \\
& +C R\left(t^{\frac{5}{52}}+t^{\frac{3}{13}}+t^{\frac{1}{2}}\right) \sup _{t \in\left[0, T_{*}\right.} t^{\frac{7}{13}}\left\|v_{1}-v_{2}\right\|_{\mathbb{H}^{2}} \\
\leq & 2 C R\left(t^{\frac{5}{52}}+t^{\frac{3}{13}}+t^{\frac{1}{2}}+t^{\frac{33}{52}}+t^{\frac{27}{26}}\right)\left(\sup _{t \in\left[0, T_{*}\right)}\left\|v_{1}-v_{2}\right\|_{\mathbb{H}^{1}}+\sup _{t \in\left[0, T_{*}\right)} t^{\frac{7}{13}}\left\|v_{1}-v_{2}\right\|_{\mathbb{H}^{2}}\right) .
\end{aligned}
$$

By (3.11) and (3.18), we can choose $T_{*}$ small enough to obtain

$$
\sup _{t \in\left[0, T_{*}\right)}\|\mathcal{L}(v)\|_{\mathbb{H}^{1}}+\sup _{t \in\left[0, T_{*}\right)} t^{\frac{7}{13}}\|\mathcal{L}(v)\|_{\mathbb{H}^{2}} \leq R
$$

and

$$
\begin{aligned}
& \sup _{t \in\left[0, T_{*}\right)}\left\|\mathcal{L}\left(v_{1}\right)-\mathcal{L}\left(v_{2}\right)\right\|_{\mathbb{H}^{1}}+\sup _{t \in\left[0, T_{*}\right)} t^{\frac{7}{13}}\left\|\mathcal{L}\left(v_{1}\right)-\mathcal{L}\left(v_{2}\right)\right\|_{\mathbb{H}^{2}} \\
< & \frac{1}{2}\left(\sup _{t \in\left[0, T_{*}\right)}\left\|v_{1}-v_{2}\right\|_{\mathbb{H}^{1}}+\sup _{t \in\left[0, T_{*}\right)} t^{\frac{7}{13}}\left\|v_{1}-v_{2}\right\|_{\mathbb{H}^{2}}\right) .
\end{aligned}
$$

By the interpolation inequalities, we deduce that

$$
\begin{aligned}
\|((v+Z) \cdot \nabla)(v+Z)\|_{\mathbb{H}^{1}} & \leq\|\nabla(v+Z)\|_{L^{4}}^{2}+\|v+Z\|_{L_{x}^{\infty}}\|v+Z\|_{\mathbb{H}^{2}} \\
& \leq C\|\nabla(v+Z)\|_{\mathbb{H}^{\frac{1}{2}}}^{\frac{1}{2}}\|v+Z\|_{\mathbb{H}^{2}}^{\frac{3}{2}}+C\|v+Z\|_{\mathbb{H}^{1}}^{\frac{1}{2}}\|v+Z\|_{\mathbb{H}^{2}}^{\frac{3}{2}} \\
& \leq C R^{\frac{1}{2}} t^{-\frac{21}{26}}\left(t^{\frac{21}{26}}\|v\|_{\mathbb{H}^{2}}^{\frac{3}{2}}+t^{\frac{21}{26}}\|Z\|_{\mathbb{H}^{2}}^{\frac{3}{2}}\right) \\
& \leq C R^{2} t^{-\frac{21}{26}}+C R^{2}
\end{aligned}
$$

which implies that

$$
\int_{0}^{t} e^{-(t-s) A}((v+Z) \cdot \nabla)(v+Z) d s \in C\left(\left[0, T_{*}\right) ; \mathbb{H}^{1}\right), \quad t \in\left[0, T_{*}\right), \quad \mathbb{P}-a . s . .
$$

Thus, for $v \in \mathcal{B}_{R}^{T_{*}}$, it holds that

$$
\mathcal{L}(v) \in C\left(\left[0, T_{*}\right) ; \mathbb{H}^{1}\right), \mathbb{P}-\text { a.s.. }
$$

By the virtue of (3.19) and (3.20), we deduce that $\mathcal{L}$ maps $\mathcal{B}_{R}^{T_{*}}$ into itself and is a strictly contraction mapping in $\mathcal{B}_{R}^{T_{*}}$. Hence, due to the contraction principle, $\mathcal{L}$ has a unique fixed point denoted by $v$ in $\mathcal{B}_{R}^{T_{*}}$, which is the unique mild solution to (3.6) on the small time interval $\left[0, T_{*}(\omega)\right)$. 


\subsection{Global existence of the strong solutions}

In the previous subsection, we have proven the existence and uniqueness of the mild solution for a short interval of time, whose length depends on the initial data. In this part, we devote to proving the global existence of a mild solution to $3 \mathrm{D}$ stochastic Burgers equations, when $u_{0}$ is $\mathcal{F}_{0}$-adapted initial value satisfying $\left\|u_{0}\right\|_{L_{x}^{\infty}} \vee\left\|u_{0}\right\|_{\mathbb{H}^{1}}<\infty$. Let us consider the mild solution that corresponds to this initial data on its maximal existence time interval $\left[0, \tau_{*}\right)$. Specifically, fixed any $\omega \in \Omega$, we will establish a priori upper estimates of this solution in the interval $\left[0, \tau_{*}\right)$. In particular, we will show $\tau_{*}=+\infty$ by obtaining the uniform $\mathbb{H}^{1}$ norm estimates of this mild solution on the time interval $\left[0, \tau_{*}\right)$. The maximum and minimum principles play a key role.

In the following, from (3.22) to (3.40), we try to establish a priori estimates for the local solution to system (1.1) in $\mathbb{H}^{1}$ space to obtain the global well-posedness of the system (1.1).

Under Hypothesis H1, for $t \in[0, T], x \in D$, it holds that

$$
W(t, x)=\sum_{k=1}^{\infty} r_{k} \beta_{k}(t)=\left(\sum_{k=1}^{\infty} \bar{e}_{k} \beta_{k}(t), 0,0\right) \in \mathbb{R}^{3} .
$$

Then, it follows that

$$
\begin{aligned}
Z(t, x) & =\int_{0}^{t} e^{-(t-s) A} G d W=\sum_{k=1}^{\infty} \sqrt{\lambda_{k}} \int_{0}^{t} e^{-(t-s) A} r_{k} d \beta_{k}(s) \\
& =\left(\sum_{k=1}^{\infty} \sqrt{\lambda_{k}} \int_{0}^{t} e^{-(t-s) A} \bar{e}_{k} d \beta_{k}(s), 0,0\right)=\left(Z_{1}, Z_{2}, Z_{3}\right) .
\end{aligned}
$$

For $t \in\left[0, \tau_{*}\right)$, denote

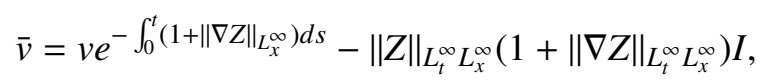

where $I=(1,1,1)$ and $\|\nabla Z\|_{L_{t}^{\infty} L_{x}^{\infty}}:=\sup _{(t, x) \in\left[0, \tau_{*}\right) \times D}|\nabla Z(t, x)| \leq \sup _{t \in\left[0, \tau_{*}\right)}\|Z(t)\|_{\mathbb{H}^{\frac{5}{2}}}$.

Substituting (3.23) into (3.6), we have

$$
\begin{aligned}
& \Delta \bar{v}+[(v+Z) \cdot \nabla] \bar{v}-\left(1+\|\nabla Z\|_{\left.L_{x}^{\infty}\right) \bar{v}+(\bar{v} \cdot \nabla) Z-\partial_{t} \bar{v}}\right. \\
& =\|Z\|_{L_{t}^{\infty} L_{x}^{\infty}}\left(1+\|\nabla Z\|_{L_{t}^{\infty} L_{x}^{\infty}}\right)\left[\left(1+\|\nabla Z\|_{L_{x}^{\infty}}\right) I-(I \cdot \nabla) Z\right]-(Z \cdot \nabla) Z e^{-\int_{0}^{t}\left(1+\|\nabla Z\|_{\left.L_{x}^{\infty}\right) d s}\right.} \\
& \geq\|Z\|_{L_{t}^{\infty} L_{x}^{\infty}}\left(1+\|\nabla Z\|_{L_{t}^{\infty} L_{x}^{\infty}}\right)-\|Z\|_{L_{x}^{\infty}}\|\nabla Z\|_{L_{x}^{\infty}}>0 .
\end{aligned}
$$

Denote by $v:=\left(v_{1}, v_{2}, v_{3}\right), \bar{v}:=\left(\bar{v}_{1}, \bar{v}_{2}, \bar{v}_{3}\right)$. Then by (3.24), we get

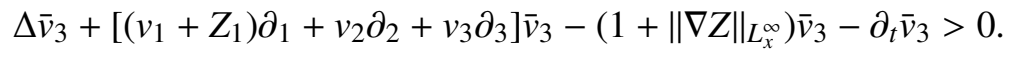

Note that under Hypothesis $\mathrm{H} 1$, the coefficient of $\bar{v}_{3}$ is $-\left(1+\|\nabla Z\|_{L_{x}^{\infty}}\right)$ instead of $-\left(1+\|\nabla Z\|_{L_{x}^{\infty}}\right)+\partial_{3} Z_{3}$, which guarantees the application of the maximum principle of parabolic equations (see Theorem 7, P174 in [40]). Then, it yields

$$
\max _{(t, x) \in\left[0, \tau_{*}\right) \times D} \bar{v}_{3}(t, x) \leq \max _{x \in D} \bar{v}_{3}(0, x),
$$


then we deduce from (3.23) that

$$
\max _{(t, x) \in\left[0, \tau_{*}\right) \times D}\left[v_{3}(t, x) e^{-\int_{0}^{t}\left(1+\|\nabla Z\|_{L_{x}^{\infty}}\right) d s}\right] \leq \max _{x \in D} v_{3}(0, x) .
$$

Thereby, we reach

$$
\begin{aligned}
& \max _{(t, x) \in\left[0, \tau_{*}\right) \times D} v_{3}(t, x) e^{-\int_{0}^{\tau_{*}}\left(1+\|\nabla Z\|_{L_{x}^{\infty}}\right) d s} \\
\leq & \max _{(t, x) \in\left[0, \tau_{*}\right) \times D}\left[v_{3}(t, x) e^{-\int_{0}^{t}\left(1+\|\nabla Z\|_{L_{x}^{\infty}}\right) d s}\right] \\
\leq & \max _{x \in D} v_{3}(0, x),
\end{aligned}
$$

which implies

$$
\max _{(t, x) \in\left[0, \tau_{*}\right) \times D} v_{3}(t, x) \leq \max _{x \in D} v_{3}(0, x) e^{\int_{0}^{\tau_{*}}\left(1+\|\nabla Z\|_{L_{x}^{\infty}}\right) d s}
$$

Moreover, set

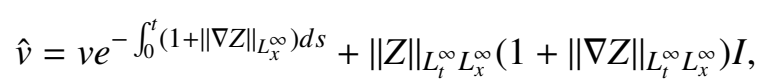

where $I=(1,1,1)$. Substituting (3.26) into (3.6), we get

$$
\begin{aligned}
& \Delta \hat{v}+[(v+Z) \cdot \nabla] \hat{v}-\left(1+\|\nabla Z\|_{L_{x}^{\infty}}\right) \hat{v}+(\hat{v} \cdot \nabla) Z-\partial_{t} \hat{v}
\end{aligned}
$$

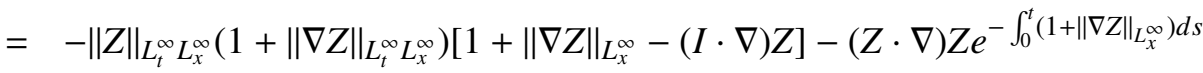

$$
\begin{aligned}
& \leq-\|Z\|_{L_{t}^{\infty} L_{x}^{\infty}}\left(1+\|\nabla Z\|_{L_{t}^{\infty} L_{x}^{\infty}}\right)+\|Z\|_{L_{x}^{\infty}}\|\nabla Z\|_{L_{x}^{\infty}}<0 \text {. }
\end{aligned}
$$

Denote by $\hat{v}=\left(\hat{v}_{1}, \hat{v}_{2}, \hat{v}_{3}\right) \in \mathbb{R}^{3}$, taking into account the fact that $Z_{3}=0$, we obtain

$$
\Delta \hat{v}_{3}+\left[\left(v_{1}+Z_{1}\right) \partial_{1}+v_{2} \partial_{2}+v_{3} \partial_{3}\right] \hat{v}_{3}-\left(1+\|\nabla Z\|_{L_{x}^{\infty}}\right) \hat{v}_{3}-\partial_{t} \hat{v}_{3}<0
$$

By the minimum principle of parabolic equations (see Theorem 7, P174 in [40]), we deduce that

$$
\min _{(t, x) \in\left[0, \tau_{*}\right) \times D} \hat{v}_{3}(t, x) \geq \min _{x \in D} \hat{v}_{3}(0, x), \text { a.s. }
$$

which implies

$$
\min _{(t, x) \in\left[0, \tau_{*}\right) \times D}\left[v_{3}(t, x) e^{-\int_{0}^{t}\left(1+\|\nabla Z\|_{L_{x}^{\infty}}\right) d s}\right] \geq \min _{x \in D} v_{3}(0, x) .
$$

Thus,

$$
\begin{aligned}
\min _{(t, x) \in\left[0, \tau_{*}\right) \times D} v_{3}(t, x) & =\min _{(t, x) \in\left[0, \tau_{*}\right) \times D} v_{3}(t, x) e^{-\int_{0}^{t}\left(1+\|\nabla Z\|_{L_{x}^{\infty}}\right) d s} e^{\int_{0}^{t}\left(1+\|\nabla Z\|_{L_{x}^{\infty}}\right) d s} \\
& \geq \min _{(t, x) \in\left[0, \tau_{*}\right) \times D} v_{3}(t, x) e^{-\int_{0}^{t}\left(1+\|\nabla Z\|_{L_{x}^{\infty}}\right) d s} e^{-\int_{0}^{\tau_{*}}\left(1+\|\nabla Z\|_{L_{x}^{\infty}}\right) d s} \\
& \geq \min _{x \in D} v_{3}(0, x) e^{-\int_{0}^{\tau_{*}}\left(1+\|\nabla Z\|_{\left.L_{x}^{\infty}\right) d s} .\right.}
\end{aligned}
$$


By (3.25) and (3.28), we conclude that

$$
\sup _{t \in\left[0, \tau_{*}\right)}\left\|v_{3}\right\|_{L_{x}^{\infty}} \leq\left\|v_{0}\right\|_{L_{x}^{\infty}} e^{\int_{0}^{\tau_{*}}\left(1+\|\nabla Z\|_{L_{x}^{\infty}}\right) d s}
$$

Similarly, taking into account the fact that $Z_{2}=0$, we also have

$$
\sup _{t \in\left[0, \tau_{*}\right)}\left\|v_{2}\right\|_{L_{x}^{\infty}} \leq\left\|v_{0}\right\|_{L_{x}^{\infty}} e^{\int_{0}^{\tau_{*}}\left(1+\|\nabla Z\|_{\left.L_{x}^{\infty}\right) d s}\right.}
$$

In the following, we will estimate $\sup _{t \in\left[0, \tau_{*}\right)}\left\|v_{1}(t)\right\|_{L_{x}^{\infty}}$. By (3.6), it follows that

$$
\begin{aligned}
& \partial_{t} v_{1}-\Delta v_{1}-\left[\left(Z_{1}+v_{1}\right) \partial_{1}+v_{2} \partial_{2}+v_{3} \partial_{3}\right] v_{1}-v_{1} \partial_{1} Z_{1} \\
= & v_{2} \partial_{2} Z_{1}+v_{3} \partial_{3} Z_{1}+Z_{1} \partial_{1} Z_{1} .
\end{aligned}
$$

Let

$$
\tilde{v}_{1}:=v_{1} e^{-\int_{0}^{t}\left(1+\|\nabla Z\|_{L_{x}^{\infty}}\right) d s}-\left(\|Z\|_{L_{t}^{\infty} L_{x}^{\infty}}+\left\|v_{2}\right\|_{L_{t}^{\infty} L_{x}^{\infty}}+\left\|v_{3}\right\|_{L_{t}^{\infty} L_{x}^{\infty}}\right)\|\nabla Z\|_{L_{t}^{\infty} L_{x}^{\infty}} .
$$

Substituting (3.32) into (3.31), we get

$$
\begin{aligned}
& \Delta \tilde{v}_{1}+\left[\left(Z_{1}+v_{1}\right) \partial_{1}+v_{2} \partial_{2}+v_{3} \partial_{3}\right] \tilde{v}_{1}-\left(1+\|\nabla Z\|_{L_{x}^{\infty}}-\partial_{1} Z_{1}\right) \tilde{v}_{1}-\partial_{t} \tilde{v}_{1} \\
& =\left(1+\|\nabla Z\|_{\left.L_{x}^{\infty}\right)}\|\nabla Z\|_{L_{t}^{\infty} L_{x}^{\infty}}\left(\|Z\|_{L_{t}^{\infty} L_{x}^{\infty}}+\left\|v_{2}\right\|_{L_{t}^{\infty} L_{x}^{\infty}}+\left\|v_{3}\right\|_{L_{t}^{\infty} L_{x}^{\infty}}\right)\right. \\
& -\|\nabla Z\|_{L_{t}^{\infty} L_{x}^{\infty}}\left(\|Z\|_{L_{t}^{\infty} L_{x}^{\infty}}+\left\|v_{2}\right\|_{L_{t}^{\infty} L_{x}^{\infty}}+\left\|v_{3}\right\|_{L_{t}^{\infty} L_{x}^{\infty}}\right) \partial_{1} Z_{1} \\
& +\left(v_{2} \partial_{2} Z_{1}+v_{3} \partial_{3} Z_{1}+Z_{1} \partial_{1} Z_{1}\right) e^{-\int_{0}^{t}\left(1+\|\nabla Z\|_{L_{x}^{\infty}}\right) d s} \\
& \geq\|\nabla Z\|_{L_{t}^{\infty} L_{x}^{\infty}}\left(\|Z\|_{L_{t}^{\infty} L_{x}^{\infty}}+\left\|v_{2}\right\|_{L_{t}^{\infty} L_{x}^{\infty}}+\left\|v_{3}\right\|_{L_{t}^{\infty} L_{x}^{\infty}}\right)-\left\|v_{2}\right\|_{L_{x}^{\infty}}\|\nabla Z\|_{L_{x}^{\infty}} \\
& -\left\|v_{3}\right\|_{L_{x}^{\infty}}\|\nabla Z\|_{L_{x}^{\infty}}-\|Z\|_{L_{x}^{\infty}}\|\nabla Z\|_{L_{x}^{\infty}} \\
& >0 \text {. }
\end{aligned}
$$

By the maximum principle for parabolic equations (see Theorem 7, P174 in [40]), we have

$$
\max _{(t, x) \in\left[0, \tau_{*}\right) \times D} \tilde{v}_{1}(t, x) \leq \max _{x \in D} \tilde{v}_{1}(0, x)
$$

which implies that

$$
\max _{(t, x) \in\left[0, \tau_{*}\right) \times D} v_{1}(t, x) \leq \max _{x \in D} v_{1}(0, x) e^{\int_{0}^{\tau_{*}}\left(1+\|\nabla Z\|_{L_{x}^{\infty}}\right) d s} .
$$

Let

$$
\check{v}_{1}=v_{1} e^{-\int_{0}^{t}\left(1+\|\nabla Z\|_{\left.L_{x}^{\infty}\right) d s}\right.}+\left(\|Z\|_{L_{t}^{\infty} L_{x}^{\infty}}+\left\|v_{2}\right\|_{L_{t}^{\infty} L_{x}^{\infty}}+\left\|v_{3}\right\|_{L_{t}^{\infty} L_{x}^{\infty}}\right)\|\nabla Z\|_{L_{t}^{\infty} L_{x}^{\infty}} .
$$

Substituting (3.33) into (3.6), we deduce that

$$
\Delta \check{v}_{1}+\left[\left(Z_{1}+v_{1}\right) \partial_{1}+v_{2} \partial_{2}+v_{3} \partial_{3}\right] \check{v}_{1}-\check{v}_{1}\left(1+\|\nabla Z\|_{L_{x}^{\infty}}-\partial_{1} Z_{1}\right)-\partial_{t} \check{v}_{1}<0
$$


By the minimum principle for parabolic equations, we have

$$
\min _{(t, x) \in\left[0, \tau_{*}\right) \times D} \check{v}_{1}(t, x) \geq \min _{x \in D} \check{v}_{1}(0, x),
$$

which implies that

$$
\min _{(t, x) \in\left[0, \tau_{*}\right) \times D} v_{1}(t, x) \geq \min _{x \in D} v_{1}(0, x) e^{-\int_{0}^{\tau_{*}}\left(1+\|\nabla Z\|_{L_{x}^{\infty}}\right) d s} .
$$

Combining the conclusions about $v_{1}$, we arrive at

$$
\sup _{t \in\left[0, \tau_{*}\right)}\left\|v_{1}(t)\right\|_{L_{x}^{\infty}} \leq\|v(0)\|_{L_{x}^{\infty}} e^{\int_{0}^{\tau_{*}}\left(1+\|\nabla Z\|_{L_{x}^{\infty}}\right) d s} .
$$

Combining (3.29), (3.30) and (3.34), we deduce that

$$
\begin{aligned}
\sup _{t \in\left[0, \tau_{*}\right)}\|v(t)\|_{L_{x}^{\infty}} & \leq\left\|u_{0}\right\|_{L_{x}^{\infty}} e^{\int_{0}^{\tau_{*}}\left(1+\|\nabla Z\|_{L_{x}^{\infty}}\right) d s} \leq\left\|u_{0}\right\|_{L_{x}^{\infty}} e^{\int_{0}^{\tau_{*}}\left(1+\|Z\|_{H^{\frac{5}{2}}}\right) d s} \\
& \leq C\left(\tau_{*},\left\|u_{0}\right\|_{L_{x}^{\infty}}\right):=C_{1} .
\end{aligned}
$$

In the following, we aim to establish $\mathbb{H}^{1}$-norm estimates of $v$ based on (3.35) and Proposition 2.1. Taking the inner product with $v$ in (3.6), we get

$$
\|v(t)\|_{\mathbb{H}}^{2}+2 \int_{0}^{t}\|v(s)\|_{\mathbb{H}^{1}}^{2} d s \leq\left\|u_{0}\right\|_{\mathbb{H}}^{2}+2 \int_{0}^{t}|\langle B(v+Z, v+Z), v\rangle| d s .
$$

Note that, the last term of (3.36) can be written as

$$
\begin{aligned}
\langle B(v+Z, v+Z), v\rangle= & \left\langle\left(v_{1}+Z_{1}\right) \partial_{1}\left(v_{1}+Z_{1}\right), v_{1}\right\rangle+\left\langle v_{2} \partial_{2}\left(v_{1}+Z_{1}\right), v_{1}\right\rangle \\
& +\left\langle v_{3} \partial_{3}\left(v_{1}+Z_{1}\right), v_{1}\right\rangle+\left\langle\left(v_{1}+Z_{1}\right) \partial_{1} v_{2}, v_{2}\right\rangle \\
& +\left\langle v_{2} \partial_{2} v_{2}, v_{2}\right\rangle+\left\langle v_{3} \partial_{3} v_{2}, v_{2}\right\rangle+\left\langle\left(v_{1}+Z_{1}\right) \partial_{1} v_{3}, v_{3}\right\rangle \\
& +\left\langle v_{2} \partial_{2} v_{3}, v_{3}\right\rangle+\left\langle v_{3} \partial_{3} v_{3}, v_{3}\right\rangle \\
= & \sum_{i=1}^{9} J_{i} .
\end{aligned}
$$

Clearly, it holds that

$$
J_{1}=\left\langle v_{1} \partial_{1} v_{1}, v_{1}\right\rangle+\left\langle Z_{1} \partial_{1} v_{1}, v_{1}\right\rangle+\left\langle v_{1} \partial_{1} Z_{1}, v_{1}\right\rangle+\left\langle Z_{1} \partial_{1} Z_{1}, v_{1}\right\rangle
$$

By the boundary condition, it yields

$$
\left\langle v_{1} \partial_{1} v_{1}, v_{1}\right\rangle=\int_{D}\left(v_{1}\right)^{2} \partial_{1} v_{1} d x=\int_{D} \partial_{1}\left[\frac{\left(v_{1}\right)^{3}}{3}\right] d x=0 .
$$

By the same method, we deduce that $J_{5}=J_{9}=0$. 
By Hölder inequality and (3.7), we get

$$
\begin{aligned}
& \left\langle Z_{1} \partial_{1} v_{1}, v_{1}\right\rangle \leq C\left\|Z_{1}\right\|_{L_{x}^{\infty}}\left\|v_{1}\right\|_{\mathbb{H}^{1}}\left\|v_{1}\right\|_{\mathbb{H}} \leq C\left\|Z_{1}\right\|_{\mathbb{H}^{1}}^{\frac{1}{2}}\left\|Z_{1}\right\|_{\mathbb{H}^{2}}^{\frac{1}{2}}\left\|v_{1}\right\|_{\mathbb{H}^{1}}\left\|v_{1}\right\|_{\mathbb{H}}, \\
& \left\langle v_{1} \partial_{1} Z_{1}, v_{1}\right\rangle \leq C\left\|v_{1}\right\|_{L^{2}}\left\|v_{1}\right\|_{L^{3}}\left\|\partial_{1} Z_{1}\right\|_{L^{6}} \leq C\left\|Z_{1}\right\|_{\mathbb{H}^{2}}\left\|v_{1}\right\|_{\mathbb{H}^{\frac{1}{2}}}^{\frac{3}{2}}\left\|v_{1}\right\|_{\mathbb{H}^{1}}^{\frac{1}{2}}, \\
& \left\langle Z_{1} \partial_{1} Z_{1}, v_{1}\right\rangle \leq C\left\|v_{1}\right\|_{L^{2}}\left\|Z_{1}\right\|_{L^{3}}\left\|\partial_{1} Z_{1}\right\|_{L^{6}} \leq C\left\|Z_{1}\right\|_{\mathbb{H}^{2}}\left\|Z_{1}\right\|_{\mathbb{H}^{2}}^{\frac{1}{2}}\left\|Z_{1}\right\|_{\mathbb{H}^{1}}^{\frac{1}{2}}\left\|v_{1}\right\|_{\mathbb{H}} .
\end{aligned}
$$

Combining all the previous estimates and by Proposition 2.1, we conclude that

$$
\begin{aligned}
\int_{0}^{t} J_{1}(s) d s \leq & C \sup _{t \in\left[0, \tau_{*}\right)}\left(\left\|Z_{1}\right\|_{\mathbb{H}^{1}}^{\frac{1}{2}}\left\|Z_{1}\right\|_{\mathbb{H}^{2}}^{\frac{1}{2}}\right) \int_{0}^{t}\left\|v_{1}\right\|_{\mathbb{H}^{1}}\left\|v_{1}\right\|_{\mathbb{H}} d s+C \sup _{t \in\left[0, \tau_{*}\right)}\left\|Z_{1}\right\|_{\mathbb{H}^{2}} \int_{0}^{t}\left\|v_{1}\right\|_{\mathbb{H}^{2}}^{\frac{3}{2}}\left\|v_{1}\right\|_{\mathbb{H}^{1}}^{\frac{1}{2}} d s \\
& +C \sup _{t \in\left[0, \tau_{*}\right)}\left(\left\|Z_{1}\right\|_{\mathbb{H}^{2}}\left\|Z_{1}\right\|_{\mathbb{H}^{2}}^{\frac{1}{2}}\left\|Z_{1}\right\|_{\mathbb{H}^{1}}^{\frac{1}{2}}\right) \int_{0}^{t}\left\|v_{1}\right\|_{\mathbb{H}} d s \\
\leq & \frac{1}{18} \int_{0}^{t}\left\|v_{1}\right\|_{\mathbb{H}^{1}}^{2} d s+C \int_{0}^{t}\left(1+\left\|v_{1}\right\|_{\mathbb{H}}^{2}\right) d s .
\end{aligned}
$$

Similarly, by (3.35), we get

$$
\begin{aligned}
\int_{0}^{t} J_{2}(s) d s & \leq \int_{0}^{t}\left|\left\langle v_{2} \partial_{2} v_{1}, v_{1}\right\rangle\right| d s+\int_{0}^{t}\left|\left\langle v_{2} \partial_{2} Z_{1}, v_{1}\right\rangle\right| d s \\
& \leq C\left\|v_{1}\right\|_{L_{t}^{\infty} L_{x}^{\infty}} \int_{0}^{t}\left\|v_{2}\right\|_{\mathbb{H}}\left(\left\|v_{1}\right\|_{\mathbb{H}^{1}}+\left\|Z_{1}\right\|_{\mathbb{H}^{1}}\right) d s \\
& \leq \frac{1}{18} \int_{0}^{t}\left\|v_{1}\right\|_{\mathbb{H}^{1}}^{2} d s+C\left(C_{1}\right) \int_{0}^{t}\left(1+\left\|v_{2}\right\|_{\mathbb{H}}^{2}\right) d s
\end{aligned}
$$

and

$$
\begin{aligned}
& \int_{0}^{t} J_{3}(s) d s \leq C\left\|v_{3}\right\|_{L_{t}^{\infty} L_{x}^{\infty}} \int_{0}^{t}\left(\left\|v_{1}\right\|_{\mathbb{H}^{1}}+\left\|Z_{1}\right\|_{\mathbb{H}^{1}}\right)\left\|v_{1}\right\|_{\mathbb{H}} d s \leq \frac{1}{18} \int_{0}^{t}\left\|v_{1}\right\|_{\mathbb{H}^{1}}^{2} d s+C\left(C_{1}\right) \int_{0}^{t}\left(1+\left\|v_{1}\right\|_{\mathbb{H}}^{2}\right) d s, \\
& \int_{0}^{t} J_{4}(s) d s \leq C\left\|v_{2}\right\|_{L_{t}^{\infty}} L_{x}^{\infty} \int_{0}^{t}\left(\left\|v_{1}\right\|_{\mathbb{H}}+\left\|Z_{1}\right\|_{\mathbb{H}}\right)\left\|v_{2}\right\|_{\mathbb{H}^{1}} d s \leq \frac{1}{18} \int_{0}^{t}\left\|v_{2}\right\|_{\mathbb{H}^{1}}^{2} d s+C\left(C_{1}\right) \int_{0}^{t}\left(1+\left\|v_{1}\right\|_{\mathbb{H}}^{2}\right) d s, \\
& \int_{0}^{t} J_{6}(s) d s \leq C\left\|v_{3}\right\|_{L_{t}^{\infty} L_{x}^{\infty}} \int_{0}^{t}\left\|v_{2}\right\|_{\mathbb{H}^{1}}\left\|v_{2}\right\|_{\mathbb{H}} d s \leq \frac{1}{18} \int_{0}^{t}\left\|v_{2}\right\|_{\mathbb{H}^{1}}^{2} d s+C\left(C_{1}\right) \int_{0}^{t}\left\|v_{2}\right\|_{\mathbb{H}}^{2} d s, \\
& \int_{0}^{t} J_{7}(s) d s \leq C\left\|v_{3}\right\|_{L_{t}^{\infty} L_{x}^{\infty}} \int_{0}^{t}\left\|v_{3}\right\|_{\mathbb{H}^{1}}\left(\left\|v_{1}\right\|_{\mathbb{H}}+\left\|Z_{1}\right\|_{\mathbb{H}}\right) d s \leq \frac{1}{18} \int_{0}^{t}\left\|v_{3}\right\|_{\mathbb{H}^{1}}^{2} d s+C\left(C_{1}\right) \int_{0}^{t}\left(1+\left\|v_{1}\right\|_{\mathbb{H}}^{2}\right) d s, \\
& \int_{0}^{t} J_{8}(s) d s \leq C\left\|v_{2}\right\|_{L_{t}^{\infty} L_{x}^{\infty}} \int_{0}^{t}\left\|v_{3}\right\|_{\mathbb{H}_{1}}\left\|v_{3}\right\|_{\mathbb{H}} d s \leq \frac{1}{18} \int_{0}^{t}\left\|v_{3}\right\|_{\mathbb{H}^{1}}^{2} d s+C\left(C_{1}\right) \int_{0}^{t}\left\|v_{3}\right\|_{\mathbb{H}}^{2} d s .
\end{aligned}
$$

Based on the above estimates, we conclude that

$$
\|v(t)\|_{\mathbb{H}^{\mathbb{1}}}^{2}+\int_{0}^{t}\|v(s)\|_{\mathbb{H}^{1}}^{2} d s \leq\left\|u_{0}\right\|_{\mathbb{H}}^{2}+C\left(C_{1}\right) \int_{0}^{t}\left(1+\|v(s)\|_{\mathbb{H}^{\prime}}^{2}\right) d s .
$$

By Gronwall inequality, we get

$$
\sup _{t \in\left[0, \tau_{*}\right)}\|v(t)\|_{\mathbb{H}}+\int_{0}^{\tau_{*}}\|v(t)\|_{\mathbb{H}^{1}}^{2} d t \leq\left(\left\|u_{0}\right\|_{\mathbb{H}}^{2}+C\left(C_{1}, \tau_{*}\right)\right) e^{C\left(C_{1}, \tau_{*}\right)}:=C_{2}\left(\tau_{*},\left\|u_{0}\right\|_{L_{x}^{\infty}},\left\|u_{0}\right\|_{\mathbb{H}}\right) .
$$


Multiplying (3.6) by $A v$ and integrating over $D$, we have

$$
\|v(t)\|_{\mathbb{H}^{1}}^{2}+2 \int_{0}^{t}\|v(s)\|_{\mathbb{H}^{2}}^{2} d s \leq\left\|u_{0}\right\|_{\mathbb{H}^{1}}^{2}+2 \int_{0}^{t}|\langle B(v+Z, v+Z), A v\rangle| d s
$$

The last term of (3.39) can be rewritten as

$$
\begin{aligned}
\langle B(v+Z, v+Z), A v\rangle= & \left\langle\left(v_{1}+Z_{1}\right) \partial_{1}\left(v_{1}+Z_{1}\right), A v_{1}\right\rangle+\left\langle v_{2} \partial_{2}\left(v_{1}+Z_{1}\right), A v_{1}\right\rangle \\
& +\left\langle v_{3} \partial_{3}\left(v_{1}+Z_{1}\right), A v_{1}\right\rangle+\left\langle\left(v_{1}+Z_{1}\right) \partial_{1} v_{2}, A v_{2}\right\rangle \\
& +\left\langle v_{2} \partial_{2} v_{2}, A v_{2}\right\rangle+\left\langle v_{3} \partial_{3} v_{2}, A v_{2}\right\rangle \\
& +\left\langle\left(v_{1}+Z_{1}\right) \partial_{1} v_{3}, A v_{3}\right\rangle+\left\langle v_{2} \partial_{2} v_{3}, A v_{3}\right\rangle \\
& +\left\langle v_{3} \partial_{3} v_{3}, A v_{3}\right\rangle .
\end{aligned}
$$

Similar to the above, we get

$$
2 \int_{0}^{t}|\langle B(v+Z, v+Z), A v\rangle| d s \leq \int_{0}^{t}\|v(s)\|_{\mathbb{H}^{2}}^{2} d s+C\left(C_{1}\right) \int_{0}^{t}\left(1+\|v(s)\|_{\mathbb{H}^{1}}^{2}\right) d s .
$$

Hence,

$$
\|v(t)\|_{\mathbb{H}^{1}}^{2}+\int_{0}^{t}\|v(s)\|_{\mathbb{H}^{2}}^{2} d s \leq\left\|u_{0}\right\|_{\mathbb{H}^{1}}^{2}+C\left(C_{1}\right) \int_{0}^{t}\left(1+\|v(s)\|_{\mathbb{H}^{1}}^{2}\right) d s
$$

By Gronwall inequality, we get

$$
\begin{aligned}
\sup _{t \in\left[0, \tau_{*}\right)}\|v(t)\|_{\mathbb{H}^{1}}^{2}+\int_{0}^{\tau_{*}}\|v(s)\|_{\mathbb{H}^{2}}^{2} d s & \leq\left(\left\|u_{0}\right\|_{\mathbb{H}^{1}}^{2}+C\left(\tau_{*}, C_{1}\right)\right) e^{C\left(\tau_{*}, C_{1}\right)} \\
& :=C_{3}\left(\tau_{*},\left\|u_{0}\right\|_{L_{x}^{\infty}},\left\|u_{0}\right\|_{\mathbb{H}^{1}}\right)<\infty \quad \mathbb{P}-\text { a.s.. }
\end{aligned}
$$

Now, we are ready to give the proof of Theorem 3.1.

Proof of Theorem 3.1. Based on the above discussion, it suffices to prove the global existence of mild solution to (3.6). Indeed, for any $\omega \in \Omega$, let $v(\omega)$ be the unique mild solution to (3.6) on the maximal interval $\left[0, \tau_{*}(\omega)\right)$. If for $\mathbb{P}$-a.s. $\omega \in \Omega$, it holds that $\tau_{*}(\omega)=+\infty$, then we complete the proof. Otherwise, there exists a set $\mathcal{N}$ whose measure is nonzero and for all $\omega \in \mathcal{N}$, it satisfies $\tau_{*}(\omega)<+\infty$. In this case, for all $\omega \in \mathcal{N}$, it holds that $\lim \sup _{t \rightarrow \tau_{*}}\|v(t, \omega)\|_{\mathbb{H}^{1}}=+\infty$, which is impossible from (3.40). Thus, we have $\tau_{*}=+\infty, \mathbb{P}-$ a.s..

Let $u_{1}$ and $u_{2}$ be two solutions of (2.2) with initial data $u_{1}(0)$ and $u_{2}(0)$, respectively. Denote by $u=u_{1}-u_{2}$ and $u_{0}=u_{1}(0)-u_{2}(0)$. Then we have

$$
\partial_{t} u+A u=B\left(u, u_{1}\right)+B\left(u_{2}, u\right) .
$$

Multiplying $A u$ in (3.41) and integrating with respect to spatial variable, it yields

$$
\begin{aligned}
\frac{d\|u(t)\|_{\mathbb{H}^{1}}^{2}}{d t}+2\|A u\|_{\mathbb{H}}^{2} & \leq 2\left|\left(A u, B\left(u, u_{1}\right)\right)\right|+2\left|\left(A u, B\left(u_{2}, u\right)\right)\right| \\
& \leq C\|A u\|_{L^{2}}\left\|\nabla u_{1}\right\|_{L^{2}}\|u\|_{L_{x}^{\infty}}+C\|A u\|_{L^{2}}\|\nabla u\|_{L^{2}}\left\|u_{2}\right\|_{L_{x}^{\infty}} .
\end{aligned}
$$


By (3.7), it follows that

$$
C\|A u\|_{L^{2}}\left\|\nabla u_{1}\right\|_{L^{2}}\|u\|_{L_{x}^{\infty}} \leq C\left\|u_{1}\right\|_{\mathbb{H}^{1}}\|u\|_{\mathbb{H}^{1}}^{\frac{1}{2}}\|u\|_{\mathbb{H}^{2}}^{\frac{3}{2}},
$$

and

$$
C\|A u\|_{L^{2}}\|\nabla u\|_{L^{2}}\left\|u_{2}\right\|_{L_{x}^{\infty}} \leq C\|A u\|_{\mathbb{H}}\|u\|_{\mathbb{H}^{1}}\left\|u_{2}\right\|_{\mathbb{H}^{1}}^{\frac{1}{2}}\left\|u_{2}\right\|_{\mathbb{H}^{2}}^{\frac{1}{2}},
$$

hence, by the Young inequality, we deduce that

$$
\begin{aligned}
\|u(t)\|_{\mathbb{H}^{1}}^{2}+\int_{0}^{t}\|A u\|_{\mathbb{H}}^{2} d s & \leq\left\|u_{0}\right\|_{\mathbb{H}^{1}}^{2}+C \int_{0}^{t}\left\|u_{1}\right\|_{\mathbb{H}^{1}}^{4}\|u\|_{\mathbb{H}^{1}}^{2} d s+C \int_{0}^{t}\|u\|_{\mathbb{H}^{1}}^{2}\left\|u_{2}\right\|_{\mathbb{H}^{1}}\left\|u_{2}\right\|_{\mathbb{H}^{2}} d s \\
& \leq C \int_{0}^{t}\left(\left\|u_{1}\right\|_{\mathbb{H}^{1}}^{4}+\left\|u_{2}\right\|_{\mathbb{H}^{1}}\left\|u_{2}\right\|_{H^{2}}\right)\|u\|_{\mathbb{H}^{1}}^{2} d s .
\end{aligned}
$$

Applying the Gronwall inequality, we get

$$
\sup _{t \in[0, T]}\|u(t)\|_{\mathbb{H}^{1}}^{2}+\int_{0}^{T}\|A u(s)\|_{\mathbb{H}^{2}}^{2} d s \leq\left\|u_{0}\right\|_{\mathbb{H}^{1}}^{2} \exp \left\{C \int_{0}^{T}\left(\left\|u_{1}\right\|_{\mathbb{H}^{1}}^{4}+\left\|u_{2}\right\|_{\mathbb{H}^{1}}\left\|u_{2}\right\|_{\mathbb{H}^{2}}\right) d s\right\} .
$$

Since $u_{i} \in C\left([0, T] ; \mathbb{H}^{1}\right) \cap L^{2}\left([0, T] ; \mathbb{H}^{2}\right), i=1,2$, we obtain that $u(t)$ is Lipschitz continuous with respect to the initial data $u_{0}$ in the space $\mathbb{H}^{1}$. We therefore complete the proof.

\section{Large deviations for 3D Burgers equations}

In this part, we aim to prove large deviations for the strong solutions to 3D stochastic Burgers equations. Let $\mathcal{L}\left(K_{1} ; K_{2}\right)$ (resp. $\left.\mathcal{L}_{2}\left(K_{1} ; K_{2}\right)\right)$ be the space of bounded (resp. Hilbert-Schmidt) linear operators from the Hilbert space $K_{1}$ to $K_{2}$, whose norm is denoted by $\|\cdot\|_{\mathcal{L}\left(K_{1} ; K_{2}\right)}\left(\|\cdot\|_{\mathcal{L}_{2}\left(K_{1} ; K_{2}\right)}\right)$.

Recall that $W$ is an $\mathbb{H}$-cylindrical Wiener process defined on $(\Omega, \mathcal{F}, \mathbb{P})$ with the form $W(t)=$ $\sum_{k \geq 1} \beta_{k}(t) r_{k}, t \in[0, T]$, where $r_{k}:=\left(\bar{e}_{k}, 0,0\right), k=1,2, \cdots$ with $\left\{\bar{e}_{k}\right\}_{k=1,2, \cdots}$ being the eigenbasis of $A$ in the space $L^{2}\left(D ; \mathbb{R}^{1}\right)$ and its associated eigenvalues is denoted by $\left\{\alpha_{k}\right\}_{k=1,2, \ldots}$. We assume that $G W(t)$ has the following representative:

$$
G W(t, x)=\sum_{k=1}^{\infty} \sqrt{\lambda_{k}} r_{k}(x) \beta_{k}(t)=\left(\sum_{k=1}^{\infty} \sqrt{\lambda_{k}} \bar{e}_{k}(x) \beta_{k}(t), 0,0\right) \in \mathbb{R}^{3} \text {, a.e. } x \in D,
$$

satisfying $\sum_{k=1}^{\infty} \lambda_{k} \alpha_{k}^{\frac{3}{2}+2 \varsigma}<\infty$ for some $\varsigma \in\left(0, \frac{1}{2}\right) . G$ is a Hilbert-Schmidt operator from $\mathbb{H}$ to $\left(\mathbb{H}^{\frac{3}{2}+2} \varsigma\right)^{3}$, i.e, $\sum_{k=1}^{+\infty}\left\|G r_{k}\right\|_{\mathbb{H}^{\frac{3}{2}+2 \varsigma}}^{2}<\infty$. Set $K:=\|G\|_{\mathcal{L}_{2}(\mathbb{H} ; \mathbb{H})}$ and $\tilde{K}:=\|G\|_{\mathcal{L}_{2}\left(\mathbb{H} ; \mathbb{H}^{1}\right)}$.

\subsection{The weak convergence approach and the main result}

We will recall the weak convergence approach developed by Budhiraja and Dupuis in [10]. Let us first recall some standard definitions and results from the large deviation theory (see e.g. [19])

Let $\left\{X^{\varepsilon}\right\}$ be a family of random variables defined on a probability space $(\Omega, \mathcal{F}, \mathbb{P})$ taking values in some Polish space $\mathcal{E}$. 
Definition 4.1. (Rate Function) A function $I: \mathcal{E} \rightarrow[0, \infty]$ is called a rate function if $I$ is lower semicontinuous. A rate function $I$ is called a good rate function if the level set $\{x \in \mathcal{E}: I(x) \leq M\}$ is compact for each $M<\infty$.

Definition 4.2. (Large Deviation Principle) The sequence $\left\{X^{\varepsilon}\right\}$ is said to satisfy a large deviation principle with rate function I if for each Borel subset $A$ of $\mathcal{E}$

$$
-\inf _{x \in A^{o}} I(x) \leq \lim _{\varepsilon \rightarrow 0} \varepsilon \log \mathbb{P}\left(X^{\varepsilon} \in A\right) \leq \limsup _{\varepsilon \rightarrow 0} \varepsilon \log \mathbb{P}\left(X^{\varepsilon} \in A\right) \leq-\inf _{x \in \bar{A}} I(x),
$$

where $A^{o}$ and $\bar{A}$ denote the interior and closure of $A$ in $\mathcal{E}$, respectively.

Suppose that $W(t)$ is an $\mathbb{H}$-cylindrical Wiener process defined on a given probability space $\left(\Omega, \mathcal{F},\left\{\mathcal{F}_{t}\right\}_{t \in[0, T]}, \mathbb{P}\right)$. The paths of $W$ take values in $C([0, T] ; Y)$, where $Y$ is another Hilbert space such that the embedding $\mathbb{H} \subset Y$ is Hilbert-Schmidt.

To state the criterion obtained by Budhiraja and Dupuis in [10], we introduce the following spaces. Set

$$
\begin{gathered}
\mathcal{A}=\left\{\phi: \phi \text { is an } \mathbb{H} \text {-valued }\left\{\mathcal{F}_{t}\right\} \text { - predictable process such that } \int_{0}^{T}|\phi(s)|_{\mathbb{H}}^{2} d s<\infty \mathbb{P} \text {-a.s. }\right\} \\
\qquad \begin{array}{c}
S_{M}=\left\{h \in L^{2}([0, T] ; \mathbb{H}): \int_{0}^{T}|h(s)|_{\mathbb{H}}^{2} d s \leq M\right\} ; \\
\mathcal{A}_{M}=\left\{\phi \in \mathcal{A}: \phi(\omega) \in S_{M}, \mathbb{P} \text {-a.s. }\right\} .
\end{array}
\end{gathered}
$$

Referring to [11], the set $S_{M}$ endowed with weak topology is a Polish space (complete separable metric space) and is a compact subspace of $L^{2}([0, T] ; \mathbb{H})$. For $\varepsilon \geq 0$, suppose $\mathcal{G}^{\varepsilon}: C([0, T], Y) \rightarrow \mathcal{E}$ is a measurable map. Set $X^{\varepsilon}=\mathcal{G}^{\varepsilon}(W)$ for $\varepsilon>0$. Consider the following conditions

Hypothesis $\mathbf{H} 2$ Let $\mathcal{G}^{0}: C([0, T] ; Y) \rightarrow \mathcal{E}$ be a measurable mapping.

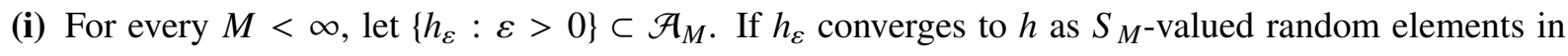
distribution, then $\mathcal{G}^{\varepsilon}\left(W(\cdot)+\frac{1}{\sqrt{\varepsilon}} \int_{0}^{\infty} h_{\varepsilon}(s) d s\right)$ converges in distribution to $\mathcal{G}^{0}\left(\int_{0}^{*} h(s) d s\right)$.

(ii) For every $M<\infty$, the set $K_{M}=\left\{\mathcal{G}^{0}\left(\int_{0} h(s) d s\right): h \in S_{M}\right\}$ is compact subset of $\mathcal{E}$.

The following result is due to Budhiraja and Dupuis in [10].

Theorem 4.1. Suppose the Hypothesis $H 2$ holds. Then $X^{\varepsilon}$ satisfies a large deviation principle on $\mathcal{E}$ with a good rate function I given by

$$
I(f)=\inf _{\left\{h \in L^{2}([0, T] ; \mathbb{H}): f=\mathcal{G}^{0}\left(\int_{0} h(s) d s\right)\right\}}\left\{\frac{1}{2} \int_{0}^{T}|h(s)|_{\mathbb{H}}^{2} d s\right\}, \quad \forall f \in \mathcal{E} .
$$

By convention, $I(\emptyset)=\infty$. 
Consider the following 3D stochastic Burgers equations driven by small noise:

$$
\left\{\begin{array}{l}
d u^{\varepsilon}(t)+A u^{\varepsilon}(t) d t=B\left(u^{\varepsilon}(t), u^{\varepsilon}(t)\right) d t+\sqrt{\varepsilon} G d W(t), \quad x \in D, t \in[0, T], \\
u^{\varepsilon}(0)=u_{0},
\end{array}\right.
$$

with $\left\|u_{0}\right\|_{L_{x}^{\infty} \vee} \vee\left\|u_{0}\right\|_{\mathbb{H}^{1}}<\infty$. According to Theorem 3.1, under Hypotheses $\mathrm{H} 0$ and $\mathrm{H} 1$, there exists a unique strong solution of (4.43) in $C\left([0, T] ; \mathbb{H}^{1}\right) \cap L^{2}\left([0, T] ; \mathbb{H}^{2}\right)$. Therefore, there exists a Borel-measurable mapping

$$
\mathcal{G}^{\varepsilon}: C([0, T], Y) \rightarrow C\left([0, T] ; \mathbb{H}^{1}\right) \cap L^{2}\left([0, T] ; \mathbb{H}^{2}\right)
$$

such that $u^{\varepsilon}(\cdot)=\mathcal{G}^{\varepsilon}(W)$.

For $h \in L^{2}([0, T] ; \mathbb{H})$, consider the following skeleton equation

$$
\left\{\begin{array}{l}
d u_{h}+A u_{h} d t=B\left(u_{h}, u_{h}\right) d t+G h(t) d t \\
u_{h}(0)=u_{0}
\end{array}\right.
$$

with $\left\|u_{0}\right\|_{L_{x}^{\infty}} \vee\left\|u_{0}\right\|_{\mathbb{H}^{1}}<\infty$. The solution $u_{h}$, whose existence will be proved in the next subsection, defines a measurable mapping $\mathcal{G}^{0}: C([0, T] ; Y) \rightarrow C\left([0, T] ; \mathbb{H}^{1}\right) \cap L^{2}\left([0, T] ; \mathbb{H}^{2}\right)$ so that $\mathcal{G}^{0}\left(\int_{0} h(s) d s\right):=u_{h}$.

Our main result of this section is

Theorem 4.2. Suppose the Hypotheses $H O$ and $H 1$ are in place and $u_{0}$ is $\mathcal{F}_{0}$-adapted initial value satisfying $\left\|u_{0}\right\|_{L_{x}^{\infty}} \vee\left\|u_{0}\right\|_{\mathbb{H}^{1}}<\infty$. Then, $u^{\varepsilon}$ satisfies a large deviation principle on $C\left([0, T] ; \mathbb{H}^{1}\right)$ with the good rate function I defined by (4.42).

The rest of the paper is devoted to the proof of this main result.

\subsection{A priori estimates of the skeleton equations}

In this subsection, we firstly show that the skeleton equation (4.44) admits a unique solution for every $h \in L^{2}([0, T] ; \mathbb{H})$ with $h(t)=\sum_{k=0}^{+\infty} h_{k}(t) r_{k}$, where $h_{k}$ is defined on $[0, T]$ and takes values in $\mathbb{R}$ for each $k(\geq 1)$. Then, we establish some properties of the solution to (4.44).

Theorem 4.3. Assume the initial value $u_{0}$ is $\mathcal{F}_{0}$-adapted satisfying $\left\|u_{0}\right\|_{\mathbb{H}^{1}}<\infty$, under Hypotheses $H 0$ and $H 1$, then for every $h \in L^{2}([0, T] ; \mathbb{H})$ with the form $h(t)=\left(\sum_{k=0}^{+\infty} \bar{e}_{k} h_{k}(t), 0,0\right) \in L^{2}\left(D ; \mathbb{R}^{3}\right)$, there exists a unique solution $u_{h}$ to equation (4.44) in the space $C\left([0, T] ; \mathbb{H}^{1}\right) \cap L^{2}\left([0, T] ; \mathbb{H}^{2}\right)$. Moreover, we have

$$
\sup _{h \in S_{M}}\left\{\sup _{t \in[0, T]}\left\|u_{h}(t)\right\|_{\mathbb{H}^{1}}^{2}+\int_{0}^{T}\left\|u_{h}(t)\right\|_{\mathbb{H}^{2}}^{2} d t\right\} \leq C\left(T, M,\left\|u_{0}\right\|_{L_{x}^{\infty}},\left\|u_{0}\right\|_{\mathbb{H}^{1}}\right):=C_{0} .
$$

Proof. Consider the following auxiliary equation:

$$
\begin{aligned}
d U(t)+A U d t & =G h(t) d t, \quad \text { on }[0, T] \times D, \\
U(t, x) & =0, \quad t \in[0, T], x \in \partial D
\end{aligned}
$$


with the initial value $U(0, x)=0$. Clearly, (4.46) has a unique solution

$$
U(t)=\int_{0}^{t} e^{-(t-s) A} G h(s) d s .
$$

We claim that under Hypothesis $\mathrm{H} 0$, the process $U(t) \in C\left([0, T] ; \mathbb{H}^{\frac{5}{2}}\right)$. Indeed, for any $0<\gamma<\gamma_{0}$, for any $t \in[0, T]$, by Hölder inequality, we deduce that

$$
\begin{aligned}
\left\|A^{\frac{5}{4}} U(t)\right\|_{\mathbb{H}}^{2} & =\int_{D}\left|\sum_{k=1}^{\infty} \int_{0}^{t} A^{\frac{5}{4}} \sqrt{\lambda_{k}} e^{-A(t-s)} r_{k}(x) h^{k}(s) d s\right|^{2} d x \\
& =\int_{D}\left|\sum_{k=1}^{\infty} \int_{0}^{t} \alpha_{k}^{\frac{5}{4}} \sqrt{\lambda_{k}} e^{-\alpha_{k}(t-s)} r_{k}(x) h^{k}(s) d s\right|^{2} d x \\
& \leq \int_{D}\left|\sum_{k=1}^{\infty}\left(\int_{0}^{t} \alpha_{k}^{\frac{5}{2}} \lambda_{k} e^{-2 \alpha_{k}(t-s)} r_{k}^{2}(x) d s\right)^{\frac{1}{2}}\left(\int_{0}^{t}\left|h^{k}(s)\right|^{2} d s\right)^{\frac{1}{2}}\right|^{2} d x \\
& \leq \int_{D}\left(\sum_{k=1}^{\infty} \int_{0}^{t} \alpha_{k}^{\frac{5}{2}} \lambda_{k} e^{-2 \alpha_{k}(t-s)} r_{k}^{2}(x) d s\right)\left(\sum_{k=1}^{\infty} \int_{0}^{t}\left|h^{k}(s)\right|^{2} d s\right) d x \\
& \leq\left(\int_{0}^{t}\|h(s)\|_{\mathbb{H}}^{2} d s\right) \int_{D} \sum_{k=1}^{\infty} r_{n}^{2}(x) \int_{0}^{t} \alpha_{k}^{\frac{5}{2}} \lambda_{k} e^{-2 \alpha_{k}(t-s)} d s d x \\
& \leq\left(\int_{0}^{t}\|h(s)\|_{\mathbb{H}}^{2} d s\right) \sum_{k=1}^{\infty} \alpha_{k}^{\frac{5}{2}} \lambda_{k} \int_{0}^{t} e^{-2 \alpha_{k}(t-s)} d s \\
& \leq C\left(\int_{0}^{t}\|h(s)\|_{\mathbb{H}}^{2} d s\right) \sum_{k=1}^{\infty} \alpha_{k}^{\frac{3}{2}} \lambda_{k}<\infty .
\end{aligned}
$$

Note that

$$
U(t)=\int_{0}^{t} e^{-(t-s) A} G h(s) d s=\left(\sum_{k=1}^{+\infty} \sqrt{\lambda_{k}} \int_{0}^{t} e^{-(t-s) A} \bar{e}_{k} h_{k}(s) d s, 0,0\right):=\left(U_{1}, U_{2}, U_{3}\right) \in \mathbb{R}^{3} .
$$

Let $V^{h}=u^{h}-U$, it satisfies

$$
d V^{h}+A V^{h} d t=B\left(V^{h}+U, V^{h}+U\right) d t
$$

Thus, using the same method as in the proof of global well-posedness of (2.2), we achieve the result.

Now, we can define $\mathcal{G}^{0}: C([0, T] ; Y) \rightarrow C\left([0, T] ; \mathbb{H}^{1}\right) \cap L^{2}\left([0, T] ; \mathbb{H}^{2}\right)$ by

$$
\mathcal{G}^{0}(\check{h}):= \begin{cases}u_{h}, & \text { if } \breve{h}=\int_{0} h(s) d s \quad \text { for some } h \in L^{2}([0, T] ; \mathbb{H}), \\ 0, & \text { otherwise. }\end{cases}
$$




\subsection{Compactness of $u_{n}$}

Let $u_{n}:=u_{h_{n}}$ be the unique strong solution of (4.44) with $h_{n} \in S_{M}$. In the following, we aim to prove the compactness of $u_{n}$. As in [25], we introduce the following space. Let $K$ be a separable Hilbert space with the norm $|\cdot|_{K}$. Given $p>1, \alpha \in(0,1)$, let $W^{\alpha, p}([0, T] ; K)$ be the Sobolev space of all $u \in L^{p}([0, T] ; K)$ such that

$$
\int_{0}^{T} \int_{0}^{T} \frac{|u(t)-u(s)|_{K}^{p}}{|t-s|^{1+\alpha p}} d t d s<\infty
$$

endowed with the norm

$$
|u|_{W^{\alpha, p}([0, T] ; K)}^{p}=\int_{0}^{T}|u(t)|_{K}^{p} d t+\int_{0}^{T} \int_{0}^{T} \frac{|u(t)-u(s)|_{K}^{p}}{|t-s|^{1+\alpha p}} d t d s .
$$

The following result can be found in [25].

Lemma 4.1. Let $B_{0} \subset B \subset B_{1}$ be Banach spaces, $B_{0}$ and $B_{1}$ reflexive, with compact embedding of $B_{0}$ in B. Let $p \in(1, \infty)$ and $\alpha \in(0,1)$ be given. Let $\Lambda$ be the space

$$
\Lambda=L^{p}\left([0, T] ; B_{0}\right) \cap W^{\alpha, p}\left([0, T] ; B_{1}\right),
$$

endowed with the natural norm. Then the embedding of $\Lambda$ in $L^{p}([0, T] ; B)$ is compact.

With the aid of Lemma 4.1 and the fact that $W^{1, p}([0, T] ; X) \subset W^{\alpha, p}([0, T] ; X)$ with $X$ be a Banach space and the given constants $\alpha$ and $p$ in Lemma4.1, we have

Proposition 4.4. Under Hypotheses $H O$ and $H 1$, the sequence $\left(u_{n}\right)_{n \in \mathbb{N}_{+}}$is compact in $L^{2}\left([0, T] ; \mathbb{H}^{1}\right)$.

Proof. From (4.44), we have

$$
\begin{aligned}
u_{n}(t) & =u_{0}-\int_{0}^{t} A u_{n}(s) d s+\int_{0}^{t} B\left(u_{n}(s), u_{n}(s)\right) d s+\int_{0}^{t} G h_{n}(s) d s \\
& :=J_{n}^{1}+J_{n}^{2}(t)+J_{n}^{3}(t)+J_{n}^{4}(t) .
\end{aligned}
$$

Clearly, $\left\|J_{n}^{1}\right\|_{\mathbb{H}} \leq C_{1}$. By (4.45), it follows that

$$
\left|J_{n}^{2}\right|_{W^{\alpha, 2}\left([0, T] ; \mathbb{H}^{-1}\right)}^{2} \leq C \int_{0}^{T}\left\|u_{n}(s)\right\|_{\mathbb{H}^{1}}^{2} d s \leq C_{2, \alpha} \quad \alpha \in\left(0, \frac{1}{2}\right) .
$$

By

$$
\|B(Y, Y)\|_{\mathbb{H}^{-1}} \leq C\|Y\|_{\mathbb{H}^{\frac{1}{2}}}^{\frac{1}{2}}\|Y\|_{\mathbb{H}^{1}}^{\frac{3}{2}},
$$

then, it gives

$$
\begin{aligned}
\left\|B\left(u_{n}, u_{n}\right)\right\|_{L^{2}\left([0, T] ; \mathbb{H}^{-1}\right)}^{2} & \leq C \int_{0}^{T}\left\|u_{n}\right\|_{\mathbb{H}}\left\|u_{n}\right\|_{\mathbb{H}^{1}}^{3} d t \\
& \leq C(T) \sup _{0 \leq t \leq T}\left\|u_{n}(t)\right\|_{\mathbb{H}} \int_{0}^{T}\left\|u_{n}(t)\right\|_{\mathbb{H}^{1}}^{3} d t \leq C\left(T, C_{0}\right) .
\end{aligned}
$$


As a result of (4.45), we obtain

$$
\left|J_{n}^{3}\right|_{W^{\alpha, 2}\left([0, T] ; \mathbb{H}^{-1}\right)}^{2} \leq C_{3, \alpha} \quad \alpha \in(0,1) .
$$

Moreover, by the Cauchy-Schwarz inequality, Hypothesis H0 and (4.45), we have

$$
\begin{aligned}
\left\|\int_{s}^{t} G h_{n}(r) d r\right\|_{\mathbb{H}}^{2} & \leq(t-s) \int_{s}^{t}\|G\|_{\mathcal{L}_{2}(\mathbb{H} ; \mathbb{H})}\left\|h_{n}(r)\right\|_{\mathbb{H}}^{2} d r \\
& \leq K(t-s) \int_{s}^{t}\left\|h_{n}(r)\right\|_{\mathbb{H}}^{2} d r,
\end{aligned}
$$

thus, applying the Fubini theorem, we get

$$
\begin{aligned}
\left|J_{n}^{4}\right|_{W^{\alpha, 2}([0, T] ; \mathbb{H})}^{2} & =\left|\int_{0}^{t} G h_{n}(s) d s\right|_{W^{\alpha, 2}([0, T] ; \mathbb{H})}^{2} \\
& =\int_{0}^{T}\left\|\int_{0}^{t} G h_{n}(s) d s\right\|_{\mathbb{H}}^{2} d t+\int_{0}^{T} \int_{0}^{T} \frac{\left\|\int_{s}^{t} G h_{n}(u) d u\right\|_{\mathbb{H}}^{2}}{|t-s|^{1+2 \alpha}} d t d s \\
& \leq C_{4, \alpha}
\end{aligned}
$$

for any $\alpha \in\left(0, \frac{1}{2}\right)$. Collecting all the previous estimates, we obtain

$$
\left|u_{n}\right|_{W^{\alpha, 2}\left([0, T] ; \mathbb{H}^{-1}\right)}^{2} \leq C_{5, \alpha}, \quad \forall \alpha \in\left(0, \frac{1}{2}\right)
$$

for some constant $C_{5, \alpha}>0$.

In view of (4.45), $u_{n}$ are bounded uniformly in $n$ in the space

$$
\Lambda:=L^{2}\left([0, T] ; \mathbb{H}^{2}\right) \cap W^{\alpha, 2}\left([0, T] ; \mathbb{H}^{-1}\right) .
$$

By Lemma 4.1, we obtain $\left(u_{n}\right)_{n \in \mathbb{Z}^{+}}$is compact in $L^{2}\left([0, T] ; \mathbb{H}^{1}\right)$.

As a result of (4.45) and Proposition 4.4, we have

Corollary 4.5. There exists a subsequence still denoted by $\left(u_{n}\right)_{n \in \mathbb{Z}^{+}}$and $\check{u} \in L^{\infty}\left([0, T] ; \mathbb{H}^{1}\right) \cap$ $L^{2}\left([0, T] ; \mathbb{H}^{1}\right) \cap L^{2}\left([0, T] ; \mathbb{H}^{2}\right)$ such that

$$
\begin{aligned}
& u_{n} \rightarrow \check{u} \text { weakly star in } L^{\infty}\left([0, T] ; \mathbb{H}^{1}\right), \\
& u_{n} \rightarrow \check{u} \text { strongly in } L^{2}\left([0, T] ; \mathbb{H}^{1}\right), \\
& u_{n} \rightarrow \check{u} \text { weakly in } L^{2}\left([0, T] ; \mathbb{H}^{2}\right) .
\end{aligned}
$$

\subsection{Property of $\check{u}$}

Throughout this subsection, we fix a sequence $\left(h_{n}\right)_{n \geq 0} \subset S_{M}$ such that $h_{n} \rightarrow h$ weakly in $L^{2}([0, T] ; \mathbb{H})$ and denote the corresponding solution of (4.44) by $u_{n}$. From Theorem 4.3 and Corollary 4.5, we know the weak star limit of $u_{n}$ exists, which is denoted by $\check{u}$. The following proposition tells that $\check{u}$ is exactly the solution of (4.44) with $h$. 
Proposition 4.6. The above ŭ satisfies

$$
\left\{\begin{array}{l}
d \check{u}(t)+A \check{u}(t) d t=B(\check{u}(t), \check{u}(t)) d t+G h(t) d t, \\
\check{u}(0)=u_{0} .
\end{array}\right.
$$

Before proving, we firstly introduce a lemma on the nonlinear term.

Lemma 4.2. Let $u_{v} \rightarrow u$ strongly in $L^{2}\left([0, T] ; \mathbb{H}^{1}\right)$ as $v \rightarrow 0$, then for $w \in \mathbb{H}^{1}$,

$$
\int_{0}^{T}\left(B\left(u_{v}(t), u_{v}(t)\right), w\right) d t \rightarrow \int_{0}^{T}(B(u(t), u(t)), w) d t \quad \text { as } v \rightarrow 0 .
$$

Proof. By the triangle inequality, we have

$$
\begin{aligned}
& \left|\int_{0}^{T}\left(B\left(u_{v}(t), u_{v}(t)\right), w\right) d t-\int_{0}^{T}(B(u(t), u(t)), w) d t\right| \\
& \leq \int_{0}^{T}\left|\left(B\left(u_{v}, u_{v}-u\right), w\right)\right| d t+\int_{0}^{T}\left|\left(B\left(u_{v}-u, u\right), w\right)\right| d t \\
& :=I_{1}+I_{2} .
\end{aligned}
$$

By Hölder inequality and interpolation inequality, we get

$$
\begin{aligned}
I_{1} & \leq \int_{0}^{T}\|w\|_{L^{6}}\left\|\nabla\left(u_{v}-u\right)\right\|_{\mathbb{H}}\left\|u_{v}\right\|_{L^{3}} d t \\
& \leq C \int_{0}^{T}\|w\|_{\mathbb{H}^{1}}\left\|u_{v}-u\right\|_{\mathbb{H}^{1}}\left\|u_{v}\right\|_{\mathbb{H}^{\frac{1}{2}}}^{\frac{1}{2}}\left\|u_{v}\right\|_{\mathbb{H}^{1}}^{\frac{1}{2}} d t \\
& \leq C T^{\frac{1}{2}}\|w\|_{\mathbb{H}^{1}} \sup _{t \in[0, T]}\left\|u_{v}\right\|_{\mathbb{H}^{1}}\left(\int_{0}^{T}\left\|u_{v}-u\right\|_{\mathbb{H}^{1}}^{2} d t\right)^{\frac{1}{2}}
\end{aligned}
$$

Similarly, it follows that

$$
\begin{aligned}
I_{2} & \leq \int_{0}^{T}\|w\|_{L^{6}}\left\|u_{v}-u\right\|_{\mathbb{H}}\|\nabla u\|_{L^{3}} d t \\
& \leq C \int_{0}^{T}\|w\|_{\mathbb{H}^{1}}\left\|u_{v}-u\right\|_{\mathbb{H}}\|u\|_{\mathbb{H}^{1}}^{\frac{1}{2}}\|u\|_{\mathbb{H}^{2}}^{\frac{1}{2}} d t \\
& \leq C\|w\|_{\mathbb{H}^{1}} \sup _{t \in[0, T]}\|u\|_{\mathbb{H}^{1}}^{\frac{1}{2}}\left(\int_{0}^{T}\left\|u_{v}-u\right\|_{\mathbb{H}^{2}}^{2} d t\right)^{\frac{1}{2}}\left(\int_{0}^{T}\|u\|_{\mathbb{H}^{2}} d t\right)^{\frac{1}{2}}
\end{aligned}
$$

Since $u_{v} \rightarrow u$ strongly in $L^{2}\left([0, T] ; \mathbb{H}^{1}\right)$, it gives $I_{1}+I_{2} \rightarrow 0$. We complete the proof.

Proof of Proposition 4.6 Denoting an orthonormal basis of $\mathbb{H}^{1}$ by $\left\{w_{j}\right\}_{j \geq 1}$. Taking a test function $\phi(t)$ a continuously differentiable on $[0, T]$ satisfying $\phi(T)=0$. From (4.44), we have

$$
\int_{0}^{T}\left(\frac{d u_{n}}{d t}, \phi(t) w_{j}\right) d t+\int_{0}^{T}\left(A u_{n}, \phi(t) w_{j}\right) d t=\int_{0}^{T}\left(B\left(u_{n}, u_{n}\right), \phi(t) w_{j}\right) d t+\int_{0}^{T}\left(G h_{n}(t), \phi(t) w_{j}\right) d t .
$$


By integration by parts, we get

$$
\begin{aligned}
& -\left(u_{0}, \phi(0) w_{j}\right)-\int_{0}^{T}\left(u_{n}(t), \phi^{\prime}(t) w_{j}\right) d t+\int_{0}^{T}\left(u_{n}(t), \phi(t) A w_{j}\right) d t \\
= & \int_{0}^{T}\left(B\left(u_{n}, u_{n}\right), \phi(t) w_{j}\right) d t+\int_{0}^{T}\left(G h_{n}(t), \phi(t) w_{j}\right) d t .
\end{aligned}
$$

Denote the above equality by symbols

$$
J_{1}+J_{2}(T)+J_{3}(T)=J_{4}(T)+J_{5}(T)
$$

Since $u_{n} \rightarrow \check{u}$ strongly in $L^{2}\left([0, T] ; \mathbb{H}^{1}\right)$ and by the Cauchy-Schwarz inequality, we obtain

$$
J_{2}(T)+J_{3}(T) \rightarrow-\int_{0}^{T}\left(\check{u}(t), \phi^{\prime}(t) w_{j}\right) d t+\int_{0}^{T}\left(\check{u}(t), \phi(t) A w_{j}\right) d t .
$$

It follows from Lemma 4.2 that

$$
J_{4}(T) \rightarrow \int_{0}^{T}\left(B(\check{u}, \breve{u}), \phi(t) w_{j}\right) d t .
$$

By the triangle inequality, we get

$$
\begin{aligned}
& \left|\int_{0}^{T}\left(G h_{n}(t), \phi(t) w_{j}\right) d t-\int_{0}^{T}\left(G h(t), \phi(t) w_{j}\right) d t\right| \\
= & \left|\int_{0}^{T}\left(G\left(h_{n}(t)-h(t)\right), \phi(t) w_{j}\right) d t\right| \\
\rightarrow & 0,
\end{aligned}
$$

where we have used $h_{n}-h \rightarrow 0$ weakly in $L^{2}([0, T] ; \mathbb{H})$. Based on the above, we have

$$
J_{5}(T) \rightarrow \int_{0}^{T}\left(G h(t), \phi(t) w_{j}\right) d t .
$$

From (4.50)-(4.52), for each $j$, we have

$$
\begin{aligned}
& -\int_{0}^{T}\left(\check{u}(t), \phi^{\prime}(t) w_{j}\right) d t+\int_{0}^{T}\left(\check{u}(t), A w_{j} \phi(t)\right) d t \\
= & \left(u_{0}, \phi(0) w_{j}\right)+\int_{0}^{T}\left(B(\check{u}, \breve{u}), \phi(t) w_{j}\right) d t+\int_{0}^{T}\left(G h(t), \phi(t) w_{j}\right) d t .
\end{aligned}
$$

Actually, (4.53) holds for any $\zeta$, which is a finite linear combination of $w_{j}$. That is

$$
\begin{aligned}
& -\int_{0}^{T}\left(\check{u}(t), \phi^{\prime}(t) \zeta\right) d t+\int_{0}^{T}(\check{u}(t), A \phi(t) \zeta) d t \\
= & \left(u_{0}, \phi(0) \zeta\right)+\int_{0}^{T}(B(\check{u}, \breve{u}), \phi(t) \zeta) d t+\int_{0}^{T}(G h(t), \phi(t) \zeta) d t .
\end{aligned}
$$

Then, we get

$$
\frac{d}{d t}(\check{u}, \zeta)+(A \check{u}, \zeta)=(B(\check{u}, \check{u}), \zeta)+(G h(t), \zeta)
$$


holds as an equality in distribution in $L^{2}\left([0, T] ; \mathbb{H}^{-1}\right)$, which is exactly (4.49).

Finally, it remains to prove $\breve{u}(0)=u_{0}$. Multiplying (4.55) with the same $\phi(t)$ as above and integrating with respect to $t$. By integration by parts, we have

$$
\begin{aligned}
& -\int_{0}^{T}\left(\check{u}(t), \phi^{\prime}(t) \zeta\right) d t+\int_{0}^{T}(\check{u}(t), A \phi(t) \zeta) d t \\
= & (\check{u}(0), \phi(0) \zeta)+\int_{0}^{T}(B(\breve{u}, \breve{u}), \phi(t) \zeta) d t+\int_{0}^{T}(G h(t), \phi(t) \zeta) d t .
\end{aligned}
$$

By comparison with (4.54), it gives $\left(\breve{u}(0)-u_{0}, \phi(0) \zeta\right)=0, \forall \zeta \in \mathbb{H}^{1}$. Choosing $\phi$ such that $\phi(0) \neq 0$, then

$$
\left(\check{u}(0)-u_{0}, \zeta\right)=0, \forall \zeta \in \mathbb{H}^{1} .
$$

We have $\breve{u}(0)=u_{0}$. We complete the proof.

In the following, we will establish the continuity of $\breve{u}$ in $\mathbb{H}^{1}$. Referring to [42], we introduce the following criterion to prove continuity.

Lemma 4.3. For $V$ and $H$ are two Hilbert spaces ( $V^{\prime}$ is the dual space of $V$ ) with $V \subset \subset H=H^{\prime} \subset V^{\prime}$, where $V \subset \subset H$ denotes $V$ is compactly embedded in $H$. If $u \in L^{2}([0, T] ; V), \frac{d u}{d t} \in L^{2}\left([0, T] ; V^{\prime}\right)$, then $u \in C([0, T] ; H)$.

Proposition 4.7. Assume Hypotheses $H O$ and $H 1$ hold, then $\check{u} \in C\left([0, T] ; \mathbb{H}^{1}\right)$.

Proof. In view of Lemma 4.3, we firstly need to prove $\frac{d \check{u}}{d t} \in L^{2}\left([0, T] ; \mathbb{H}^{-1}\right)$. From Proposition 4.6, we know $\check{u} \in L^{2}\left([0, T] ; \mathbb{H}^{2}\right) \cap L^{\infty}\left([0, T] ; \mathbb{H}^{1}\right)$ and

$$
\frac{d \check{u}}{d t}=-A \check{u}+B(\check{u}, \check{u})+G h(t) .
$$

Since $\breve{u}$ is bounded in $L^{2}\left([0, T] ; \mathbb{H}^{2}\right)$ and $A$ is continuous linear operator from $\mathbb{H}^{2}$ to $\mathbb{H}$, we deduce $A \breve{u}$ is bounded in $L^{2}([0, T] ; \mathbb{H})$. Similar to (4.48), we have

$$
\|B(\check{u}, \check{u})\|_{L^{2}\left([0, T] ; \mathbb{H}^{-1}\right)} \leq C .
$$

Moreover, it follows from Hypothesis H0 that

$$
\begin{aligned}
\|G h\|_{L^{2}([0, T] ; \mathbb{H})}^{2} & =\int_{0}^{T}\|G h(t)\|_{\mathbb{H}}^{2} d t \\
& \leq \int_{0}^{T}\|G\|_{\mathcal{L}_{2}(\mathbb{H} ; \mathbb{H})}^{2}\|h(t)\|_{\mathbb{H}}^{2} d t \\
& \leq K \int_{0}^{T}\|h(t)\|_{\mathbb{H}}^{2} d t \leq K M .
\end{aligned}
$$

As a result, we get

$$
\frac{d \check{u}}{d t} \in L^{2}\left([0, T] ; \mathbb{H}^{-1}\right) .
$$

From (4.45), we know $\check{u} \in L^{2}\left([0, T] ; \mathbb{H}^{2}\right)$, then we conclude the result by applying Lemma 4.3. 
By the uniqueness of (4.44), we have

Corollary 4.8. Assume Hypotheses $\mathrm{HO}$ and $\mathrm{Hl}$ hold, then $\breve{u}=u_{h}$, where $u_{h}$ is the unique strong solution of (4.44) with $h$.

Now, we can obtain

Theorem 4.9. Assume Hypotheses $H O$ and $H 1$ hold, then $u_{n}-\check{u} \rightarrow 0$ in $C\left([0, T] ; \mathbb{H}^{1}\right)$ as $n \rightarrow \infty$.

Proof. Recall $u_{n}:=u_{h_{n}}$ is the unique strong solution of (4.44) with $h_{n} \in S_{M}$ and $\check{u}$ be the solution of (4.49) with $h \in S_{M}$. Then, we deduce that

$$
\begin{aligned}
d\left(u_{n}-\check{u}\right)+A\left(u_{n}-\breve{u}\right) d t & =\left[B\left(u_{n}, u_{n}\right)-B(\breve{u}, \breve{u})\right] d t+G\left(h_{n}(t)-h(t)\right) d t \\
& =B\left(u_{n}-\check{u}, u_{n}\right) d t+B\left(\check{u}, u_{n}-\breve{u}\right) d t+G\left(h_{n}(t)-h(t)\right) d t .
\end{aligned}
$$

By the chain rule, we get

$$
\begin{aligned}
& \left\|u_{n}(t)-\check{u}(t)\right\|_{\mathbb{H}^{1}}^{2}+2 \int_{0}^{t}\left\|u_{n}-\check{u}\right\|_{\mathbb{H}^{2}}^{2} d s \\
\leq & 2 \int_{0}^{t}\left\langle A\left(u_{n}-\check{u}\right), B\left(u_{n}-\check{u}, u_{n}\right)\right\rangle d s+2 \int_{0}^{t}\left\langle A\left(u_{n}-\check{u}\right), B\left(\check{u}, u_{n}-\check{u}\right)\right\rangle d s \\
& +2 \int_{0}^{t}\left\langle A\left(u_{n}-\check{u}\right), G\left(h_{n}(t)-h(t)\right)\right\rangle d s \\
:= & I_{1}(t)+I_{2}(t)+I_{3}(t) .
\end{aligned}
$$

By Hölder inequality and interpolation inequality, we deduce that

$$
\begin{aligned}
I_{1}(t) & \leq 2 \int_{0}^{t}\left\|A\left(u_{n}-\check{u}\right)\right\|_{L^{2}}\left\|\nabla u_{n}\right\|_{L^{3}}\left\|u_{n}-\check{u}\right\|_{L^{6}} d s \\
& \leq C \int_{0}^{t}\left\|u_{n}-\check{u}\right\|_{\mathbb{H}^{2}}\left\|u_{n}\right\|_{\mathbb{H}^{1}}^{\frac{1}{2}}\left\|u_{n}\right\|_{\mathbb{H}^{2}}^{\frac{1}{2}}\left\|u_{n}-\check{u}\right\|_{\mathbb{H}^{1}} d s \\
& \leq \frac{1}{2} \int_{0}^{t}\left\|u_{n}-\check{u}\right\|_{\mathbb{H}^{2}}^{2} d s+C \int_{0}^{t}\left\|u_{n}\right\|_{\mathbb{H}^{1}}\left\|u_{n}\right\|_{\mathbb{H}^{2}}\left\|u_{n}-\check{u}\right\|_{\mathbb{H}^{1}}^{2} d s .
\end{aligned}
$$

Using the same method, it follows that

$$
\begin{aligned}
I_{2}(t) & \leq 2 \int_{0}^{t}\left\|A\left(u_{n}-\check{u}\right)\right\|_{L^{2}}\|\check{u}\|_{L^{\infty}}\left\|\nabla\left(u_{n}-\check{u}\right)\right\|_{L^{2}} d s \\
& \leq C \int_{0}^{t}\left\|u_{n}-\check{u}\right\|_{\mathbb{H}^{2}}\|\check{u}\|_{\mathbb{H}^{1}}^{\frac{1}{2}}\|\check{u}\|_{\mathbb{H}^{2}}^{\frac{1}{2}}\left\|u_{n}-\check{u}\right\|_{\mathbb{H}^{1}} d s \\
& \leq \frac{1}{2} \int_{0}^{t}\left\|u_{n}-\check{u}\right\|_{\mathbb{H}^{2}}^{2} d s+C \int_{0}^{t}\|\check{u}\|_{\mathbb{H}^{1}}\|\check{u}\|_{\mathbb{H}^{2}}\left\|u_{n}-\check{u}\right\|_{\mathbb{H}^{1}}^{2} d s .
\end{aligned}
$$

By Hypothesis H0, it yields

$$
\begin{aligned}
I_{3}(t) & \leq \int_{0}^{t}\left\|u_{n}-\check{u}\right\|_{\mathbb{H}^{1}}\|G\|_{\mathcal{L}_{2}\left(\mathbb{H} ; \mathbb{H}^{1}\right)}\left\|h_{n}(t)-h(t)\right\|_{\mathbb{H}} d s \\
& \leq C \tilde{K} \int_{0}^{t}\left\|u_{n}-\check{u}\right\|_{\mathbb{H}^{1}}\left\|h_{n}(t)-h(t)\right\|_{\mathbb{H}} d s \\
& \leq C \tilde{K} M^{\frac{1}{2}}\left(\int_{0}^{t}\left\|u_{n}-\check{u}\right\|_{\mathbb{H}^{1}}^{2} d s\right)^{\frac{1}{2}} .
\end{aligned}
$$


Combining all the above estimates, we get

$$
\begin{aligned}
& \left\|u_{n}(t)-\check{u}(t)\right\|_{\mathbb{H}^{1}}^{2}+\int_{0}^{t}\left\|u_{n}-\check{u}\right\|_{\mathbb{H}^{2}}^{2} d s \\
\leq & C \tilde{K} M^{\frac{1}{2}}\left(\int_{0}^{t}\left\|u_{n}-\check{u}\right\|_{\mathbb{H}^{1}}^{2} d s\right)^{\frac{1}{2}}+C \int_{0}^{t}\left(\left\|u_{n}\right\|_{\mathbb{H}^{1}}\left\|u_{n}\right\|_{\mathbb{H}^{2}}+\|\check{u}\|_{\mathbb{H}^{1}}\|\check{u}\|_{\mathbb{H}^{2}}\right)\left\|u_{n}-\check{u}\right\|_{\mathbb{H}^{1}}^{2} d s .
\end{aligned}
$$

By Gronwall inequality, we deduce that

$$
\begin{aligned}
& \sup _{t \in[0, T]}\left\|u_{n}(t)-\check{u}(t)\right\|_{\mathbb{H}^{1}}^{2}+\int_{0}^{T}\left\|u_{n}-\check{u}\right\|_{\mathbb{H}^{2}}^{2} d s \\
\leq & C \tilde{K} M^{\frac{1}{2}}\left(\int_{0}^{T}\left\|u_{n}-\check{u}\right\|_{\mathbb{H}^{1}}^{2} d s\right)^{\frac{1}{2}} \exp \left\{C \int_{0}^{T}\left(\left\|u_{n}\right\|_{\mathbb{H}^{1}}\left\|u_{n}\right\|_{\mathbb{H}^{2}}+\|\check{u}\|_{\mathbb{H}^{1}}\|\check{u}\|_{\mathbb{H}^{2}}\right) d s\right\} .
\end{aligned}
$$

By (4.45) and Corollary 4.5, we get

$$
\exp \left\{C \int_{0}^{T}\left(\left\|u_{n}\right\|_{\mathbb{H}^{1}}\left\|u_{n}\right\|_{\mathbb{H}^{2}}+\|\check{u}\|_{\mathbb{H}^{1}}\|\check{u}\|_{\mathbb{H}^{2}}\right) d s\right\} \leq C\left(T, M, C_{0}\right)<\infty .
$$

Since $u_{n}-\check{u} \rightarrow 0$ strongly in $L^{2}\left([0, T] ; \mathbb{H}^{1}\right)$, then

$$
\sup _{t \in[0, T]}\left\|u_{n}(t)-\check{u}(t)\right\|_{\mathbb{H}^{1}}^{2} \rightarrow 0 .
$$

We complete the proof.

\subsection{Proof of the main result}

Proof of Theorem 4.2 Due to Theorem 4.1, it suffices to verify the two conditions in Hypothesis H2.

Step 1 First, we show that the set $K_{M}=\left\{\mathcal{G}^{0}\left(\int_{0}^{0} h(s) d s\right): h \in S_{M}\right\}$ is compact subset of $C\left([0, T] ; \mathbb{H}^{1}\right)$, where $\mathcal{G}^{0}$ is defined in (4.47).

Let $\left\{u_{n}\right\}$ be a sequence in $K_{M}$ where $u_{n}$ corresponds to the solution of (4.44) with $h_{n} \in S_{M}$ in place of $h$. By the weak compactness of $S_{M}$ in $L^{2}([0, T] ; \mathbb{H})$, there exists a subsequence (which we still denote it by $\left\{h_{n}\right\}$ ) converging to a limit $h$ weakly in $S_{M}$. Denote by $u_{h}$ the strong solution of (4.44) with $h$. Utilizing Corollary 4.8 , it suffices to show that $u_{n} \rightarrow \check{u}$ in $C\left([0, T] ; \mathbb{H}^{1}\right)$. Thanks to Theorem 4.9, we complete the proof.

Step 2 Suppose that $\left\{h_{\varepsilon}: \varepsilon>0\right\} \subset \mathcal{A}_{M}$ for any fixed $M<\infty$ and $h_{\varepsilon}$ converge to $h$ as $S_{M}$-valued random elements in distribution. Recalling the definition of $\mathcal{G}^{\varepsilon}$ and by Girsanov's theorem, $\bar{u}_{h_{\varepsilon}}=\mathcal{G}^{\varepsilon}\left(W(\cdot)+\frac{1}{\sqrt{\varepsilon}} \int_{0}^{\cdot} h_{\varepsilon}(s) d s\right)$ solves the following equation

$$
\left\{\begin{array}{l}
d \bar{u}_{h_{\varepsilon}}(t)+A \bar{u}_{h_{\varepsilon}}(t) d t=B\left(\bar{u}_{h_{\varepsilon}}(t), \bar{u}_{h_{\varepsilon}}(t)\right) d t+G h_{\varepsilon} d t+\sqrt{\varepsilon} G d W(t), \\
\bar{u}_{h_{\varepsilon}}(0)=u_{0}
\end{array}\right.
$$

In order to transform (4.57) to a deterministic model, we introduce an auxiliary process $Z_{\varepsilon}$ written as

$$
\left\{\begin{array}{l}
d Z_{\varepsilon}(t)+A Z_{\varepsilon}(t) d t=\sqrt{\varepsilon} G d W(t) \\
Z_{\varepsilon}(0)=0
\end{array}\right.
$$


Under Hypotheses H0 and H1, by Itô formula, we have

$$
\lim _{\varepsilon \rightarrow 0} \mathbb{E}\left(\sup _{0 \leq t \leq T}\left\|Z_{\varepsilon}(t)\right\|_{\mathbb{H}^{1}}^{2}+\int_{0}^{T}\left\|Z_{\varepsilon}(t)\right\|_{\mathbb{H}^{2}}^{2} d t\right)=0 .
$$

Since $S_{M}$ is a Polish space, by the Skorohod representation theorem, we can construct a stochastic basis $\left(\Omega^{1}, \mathcal{F}^{1}, \mathbb{P}^{1}\right)$ and, on this basis, $S_{M} \otimes S_{M} \otimes C\left([0, T] ; \mathbb{H}^{1}\right) \cap L^{2}\left([0, T] ; \mathbb{H}^{2}\right)$-valued random variables processes $\left(\tilde{h}_{\varepsilon}, \tilde{h}, \tilde{Z}_{\varepsilon}\right)$ such that the joint distribution of $\left(\tilde{h}_{\varepsilon}, \tilde{Z}_{\varepsilon}\right)$ is the same as $\left(h_{\varepsilon}, Z_{\varepsilon}\right), \tilde{Z}_{\varepsilon} \rightarrow 0 \mathbb{P}^{1}-$ a.s. in $C\left([0, T] ; \mathbb{H}^{1}\right) \cap L^{2}\left([0, T] ; \mathbb{H}^{2}\right)$, the distribution of $h$ coincides with $\tilde{h}$ and $\tilde{h}_{\varepsilon} \rightarrow \tilde{h} \mathbb{P}^{1}-$ a.s. as $S_{M}$-valued random elements. Let $X_{\tilde{h}_{\varepsilon}}(t)$ be the solution of

$$
\left\{\begin{array}{l}
d X_{\tilde{h}_{\varepsilon}}(t)+A X_{\tilde{h}_{\varepsilon}}(t) d t=B\left(X_{\tilde{h}_{\varepsilon}}(t)+\tilde{Z}_{\varepsilon}, X_{\tilde{h}_{\varepsilon}}(t)+\tilde{Z}_{\varepsilon}\right) d t+G \tilde{h}_{\varepsilon} d t, \\
X_{\tilde{h}_{\varepsilon}}(0)=u_{0} .
\end{array}\right.
$$

The uniqueness of (4.60) implies that $X_{\tilde{h}_{\varepsilon}}$ has the same distribution with $\bar{u}_{h_{\varepsilon}}-Z_{\varepsilon}$.

By (4.59) and using similar arguments as Sect. 4.2, we get

$$
X_{\tilde{h}_{\varepsilon}} \rightarrow X_{\tilde{h}} \text { in } C\left([0, T] ; \mathbb{H}^{1}\right) \quad \mathbb{P}^{1} \text {-a.s., }
$$

where

$$
\left\{\begin{array}{l}
d X_{\tilde{h}}(t)+A X_{\tilde{h}}(t) d t=B\left(X_{\tilde{h}}(t), X_{\tilde{h}}(t)\right) d t+G \tilde{h} d t, \\
X_{\tilde{h}}(0)=u_{0} .
\end{array}\right.
$$

Recalling the definition of $\mathcal{G}^{0}$ and in view of $X_{\tilde{h}_{\varepsilon}}$ has the same distribution with $\bar{u}_{h_{\varepsilon}}-Z_{\varepsilon}$, we know $\bar{u}_{h_{\varepsilon}}-Z_{\varepsilon} \rightarrow u_{h}$ in distribution in $C\left([0, T] ; \mathbb{H}^{1}\right)$. Moreover, by (4.59), we obtain $Z_{\varepsilon} \rightarrow 0$ in distribution in $C\left([0, T] ; \mathbb{H}^{1}\right)$. Thus, $\bar{u}_{h_{\varepsilon}} \rightarrow u_{h}$ in distribution in $C\left([0, T] ; \mathbb{H}^{1}\right)$. We complete the proof.

Acknowledgements This work was partially supported by NNSF of China(Grant No. 11401057, 11801032), Key Laboratory of Random Complex Structures and Data Science, Academy of Mathematics and Systems Science, Chinese Academy of Sciences(No. 2008DP173182), China Postdoctoral Science Foundation funded project (No. 2018M641204), Natural Science Foundation Project of CQ (Grant No. cstc2016jcyjA0326), Fundamental Research Funds for the Central Universities(Grant No. 2018CDXYST0024, 63181314) and China Scholarship Council (Grant No.201506055003).

\section{References}

[1] R.A. Adams: Sobolev Space. New York: Academic Press, 1975.

[2] Y. Bakhtin, Burgers Equation with random boundary conditions, Proc.Amer.Math.Soc., 135(2007), 2257-2262.

[3] Y. Bakhtin, L. Li: Thermodynamic Limit for Directed Polymers and Stationary Solutions of the Burgers Equation,Communications on Pure and Applied Mathematics, Vol.LXXII(2019), 0536-0619. 
[4] Y. Bakhtin, E. Cator, and K. Khanin: Space-time stationary solutions for the Burgers equation, Journal of the American Mathematical Society, 27(2014), 193-238.

[5] L. Bertini, N. Cancrini, G. Jona-Lasinio: The stochastic Burgers equation, Comm. Math. Phys., 165 (1994), 211-232.

[6] H. Beteman: Some recent researches of the motion of fluid, Monthly Weather Rev. 43 (1915), 163170.

[7] A. Boritchev: Multidimensional potential Burgers turbulence, Commun.Math.Phys.,342(2016), 441489.

[8] M. Boué, P. Dupuis: A variational representation for certain functionals of Brownian motion. Ann. Probab. 26(1998), 1641-1659.

[9] Z. Brzezniak, B. Goldys, M. Neklyudov: Multidimensional stochastic Burgers equation, SIAM J. Math. Anal., 46 (2014), 871-889.

[10] A. Budhiraja; P. Dupuis: A variational representation for positive functionals of infinite dimensional Brownian motion, Probab. Math. Statist., 20(2000), 39-61.

[11] A. Budhiraja, P. Dupuis, V. Maroulas: Large deviations for infinite dimensional stochastic dynamical systems. Ann. Probab., 36 (2008), 1390-1420.

[12] Ton. Bui: Non-stationary Burgers flows with vanishing viscosity in bounded domains of $\mathbb{R}^{3}$, Math. Z., 145 (1975), 69-79.

[13] J.M. Burgers: Mathematical examples illustrating relations occurring in the theory of turbulent fluid motion, Verh. Nederl. Akad. Wetensch. Afd. Natuurk. Sect. 1. 17(1939), 1-53.

[14] D.H. Chambers, R.J. Adrian, P. Moin, D.S. Stewart, H.J. Sung: Karhunen-Loéve expansion of Burgers' model of turbulence, Phys. Fluids, 31(1988), 2573-2582.

[15] T. Chan: Scaling limits of Wick ordered KPZ equation, Commun. Math. Phys., 209 (2000), 671-690.

[16] G. Da Prato, A. Debussche: Stochastic Cahn-Hilliard equation, Nonlinear Anal., 26 (1996), 241263.

[17] G. Da Prato, J. Zabczyk: Stochastic equations in infinite dimensions, in "Encyclopedia of Mathematics and its Application”, Cambridge University Press, Cambridge, 1992.

[18] G. Da Prato, A. Debussche, R. Teman: Stochastic Burgers' equation, NoDEA, 1 (1994), 389-402.

[19] A. Dembo, O. Zeitouni: Large deviations techniques and applications, Jones and Bartlett, Boston, 1993. 
[20] Z. Dong: On the uniqueness of invariant measure of the Burgers equation driven by Lévy processes, J. Theoret. Probab., 21 (2008), 322-335.

[21] Z. Dong, L.H. Xu, X.C. Zhang: Exponential ergodicity of stochastic Burgers equations driven by $\alpha$-stable processes, J. Stat. Phys., 154 (2014),929-949.

[22] Z. Dong, T.G. Xu: One-dimensional stochastic Burgers equation driven by Lévy processes, Journal of Functional Analysis, 243 (2007), 631-678.

[23] P. Dupuis, R.S. Ellis: A weak convergence approach to the theory of large deviations, Wiley, New York, 1997.

[24] W. E, K. Khanin, A. Mazel, Ya. Sinai: Invariant measures for Burgers equation with stochastic forcing, Ann. of Math., 151 (2000), 877-900.

[25] F. Flandoli, D. Gatarek: Martingale and stationary solutions for stochastic Navier-Stokes equations, Probab. Theory Related Fields, 102(1995), 367-391.

[26] A.R. Forsyth: Theory of differential equations, Vol. 6, Cambridge University Press, 1906.

[27] M. Gourcy: Large deviation principle of occupation measure for stochastic Burgers equation, Ann. I. H. Poincaré-PR, 43 (2007), 441-459.

[28] D.Gomes, R.Iturriaga, K.Khanin, and P. Padilla: Viscosity limit of stationary distributions for the random forced Burgers equation, Moscow Mathematical Journal, 5(2005), 613-631.

[29] I. Gyöngy, D. Nualart: On the stochastic Burgers equation in the real line, Ann. Probab., 27 (1999), 782-802.

[30] I. Hosokawa, K. Yamamoto: Turbulence in the randomly forced one dimensional Burgers flow, J. Stat. Phys., 245 (1975), 245-272.

[31] R. Iturriaga and K. Khanin:Burgers turbulence and random Lagrangian systems, Comm.Math.Phys., 232(2003), 377-428.

[32] Dah-Teng Jeng: Forced Model Equation for Turbulence, The Physics of Fluids, 12 (1969), 20062010.

[33] K. Khanin, K. Zhang: Hyperbolicity of minimizers and regularity of viscosity solutions for a random Hamilton-Jacobi equation, Commun.Math.Phy.,355(2017), 803-837.

[34] M. Kardar, M. Parisi, J. C. Zhang: Dynamical scaling of growing interfaces, Phys. Rev. Lett., 56 (1986), 889-892. 
[35] A. Kiselev, O. A. Ladyzhenskaya: On the existence and uniqueness of the solution of the nonstationary problem for a viscous, incompressible fluid, (Russian) Izv. Akad. Nauk SSSR. Ser. Mat., 21(1957), 655-680.

[36] K. Liu: Stability of stochastic differential equations in infinite dimensions, New York: Springer Verlag, 2004.

[37] S. Mohammed, T.S. Zhang: Stochastic Burgers equation with random initial velocities: a Malliavin calculus approach, SIAM J. Math. Anal., 45 (2013), 2396-2420.

[38] L. Nirenberg: On elliptic partial differential equations, Ann. Sc. Norm. Sup. Pisa, 13(1959), 116162.

[39] A. Pazy: Semigroups of linear operators and applications to partial differential equations, Springer-Verlag. New York/Berlin/Heidelberg/Tokyo, 1983.

[40] M. H. Protter, H. F. Weinberger: Maximum principles in differential equations, Prentice-Hall, Englewood Cliffs, N.J., 1967.

[41] R. Temam: Infinite Dimensional Dynamics Systems in Mechanics and Physics, Berlin, Heidelberg, New York: Springer, 1988.

[42] R. Temam : Navier-Stokes equations and nonlinear functional analysis, Second edition. CBMSNSF Regional Conference Series in Applied Mathematics, 66. Society for Industrial and Applied Mathematics (SIAM), Philadelphia, PA, 1995.

[43] G. Wang, M. Zeng, B. Guo: Stochastic Burgers' equation driven by fractional Brownian motion, J. Math. Anal. Appl., 371 (2010), 210-222. 